ESAIM: COCV 19 (2013) 1189-1208

DOI: $10.1051 / \mathrm{cocv} / 2013051$
ESAIM: Control, Optimisation and Calculus of Variations

www.esaim-cocv.org

\title{
NASH EQUILIBRIUM PAYOFFS FOR STOCHASTIC DIFFERENTIAL GAMES WITH REFLECTION
}

\author{
QIAN LIN ${ }^{1}$
}

\begin{abstract}
In this paper, we investigate Nash equilibrium payoffs for nonzero-sum stochastic differential games with reflection. We obtain an existence theorem and a characterization theorem of Nash equilibrium payoffs for nonzero-sum stochastic differential games with nonlinear cost functionals defined by doubly controlled reflected backward stochastic differential equations.
\end{abstract}

Mathematics Subject Classification. 49L25, 60H10, 60H30, 90C39.

Received February 11, 2012. Revised September 25, 2012.

Published online August 27, 2013.

\section{INTRODUCTION}

In this paper, we study Nash equilibrium payoffs for nonzero-sum stochastic differential games whose cost functionals are defined by reflected backward stochastic differential equations (RBSDEs, for short). Fleming and Souganidis [7] were the first in a rigorous way to study zero-sum stochastic differential games. Since the pioneering work of Fleming and Souganidis [7], stochastic differential games have been investigated by many authors. Recently, Buckdahn and Li [3] generalized the results of Fleming and Souganidis [7] by using a Girsanov transformation argument and a backward stochastic differential equation (BSDE, for short) approach. The reader interested in this topic can be referred to Buckdahn, Cardaliaguet and Quincampoix [1], Buckdahn and Li [3], Fleming and Souganidis [7] and the references therein.

El Karoui, Kapoudjian, Pardoux, Peng and Quenez [5] introduced RBSDEs. By virtue of RBSDEs they gave a probabilistic representation for the solution of an obstacle problem for a nonlinear parabolic partial differential equation. This kind of RBSDEs also has many applications in finance, stochastic differential games and stochastic optimal control problem. In [6], El Karoui, Pardoux and Quenez showed that the price of an American option corresponds to the solution of a RBSDE. Buckdahn and Li [4] considered zero-sum stochastic differential games with reflection. $\mathrm{Wu}$ and $\mathrm{Yu}$ [12] studied one kind of stochastic recursive optimal control problem with the obstacle constraint for the cost functional defined by a RBSDE.

Buckdahn, Cardaliaguet and Rainer [2] studied Nash equilibrium payoffs for stochastic differential games. Recently, Lin $[9,10]$ studied Nash equilibrium payoffs for stochastic differential games whose cost functionals are defined by doubly controlled BSDEs. Lin $[9,10]$ generalizes the earlier result by Buckdahn, Cardaliaguet and Rainer [2]. In $[9,10]$, the admissible control processes can depend on events occurring before the beginning

Keywords and phrases. Backward stochastic differential equations, dynamic programming principle, Nash equilibrium payoffs, stochastic differential games.

1 Center for Mathematical Economics, Bielefeld University, Postfach 100131, 33501 Bielefeld, Germany. linqian1824@163.com 
of the stochastic differential game, thus, the cost functionals are not necessarily deterministic. Moreover, the cost functionals are defined with the help of BSDEs, and thus they are nonlinear. The objective of this paper is to generalize the above results, i.e., investigate Nash equilibrium payoffs for nonzero-sum stochastic differential games with reflection. However, different from the earlier results by Buckdahn, Cardaliaguet and Rainer [2] and Lin [9,10], we shall study Nash equilibrium payoffs for stochastic differential games with the running cost functionals defined with the help of RBSDEs. For this, we first study the properties of the value functions of stochastic differential games with reflection. In comparison with Buckdahn and Li [4], we shall study nonzero-sum stochastic differential games of the type of strategy against strategy, while Buckdahn and Li [4] considered the games of the type strategy against control. Combining the arguments in Buckdahn, Cardaliaguet and Quincampoix [1] and Buckdahn and Li [4], we can get the results in Section 4. Then we investigate Nash equilibrium payoffs for stochastic differential games with reflection. Our results generalizes the results in Lin [9] to the obstacle constraint case. In Lin [9], the cost functionals of both players do not have any obstacle constraint, so our results in Section 5 are more general. The proof of our results is mainly based on the techniques of mathematical analysis and the properties of BSDEs with reflection. The presence of the obstacle constraint adds us the difficulty of estimates and a supplementary complexity.

The paper is organized as follows. In Section 2, we introduce some notations and present some preliminary results concerning reflected backward stochastic differential equations, which we will need in what follows. In Section 3, we introduce nonzero-sum stochastic differential games with reflection and obtain the associated dynamic programming principle. In Section 4 we give a probabilistic interpretation of systems of Isaacs equations with obstacle. In Section 5, we obtain the main results of this paper, i.e., an existence theorem and a characterization theorem of Nash equilibrium payoffs for nonzero-sum stochastic differential games with reflection. In Section 6, we give the Proof of Theorem 4.2.

\section{PRELiminaries}

The objective of this Section is to recall some results about RBSDEs, which are useful in what follows. Let $B=\left\{B_{t}, 0 \leq t \leq T\right\}$ be a $d$-dimensional standard Brownian motion defined on a probability space $(\Omega, \mathcal{F}, \mathbb{P})$. The filtration $\mathbb{F}=\left\{\mathcal{F}_{t}, 0 \leq t \leq T\right\}$ is generated by $B$ and augmented by all $\mathbb{P}$-null sets, i.e.,

$$
\mathcal{F}_{t}=\sigma\left\{B_{r}, 0 \leq r \leq t\right\} \vee \mathcal{N}_{\mathbb{P}},
$$

where $\mathcal{N}_{\mathbb{P}}$ is the set of all $\mathbb{P}$-null sets. Let us introduce some spaces:

$$
\begin{aligned}
& L^{2}\left(\Omega, \mathcal{F}_{T}, \mathbb{P} ; \mathbb{R}^{n}\right)=\left\{\xi \mid \xi: \Omega \rightarrow \mathbb{R}^{n} \text { is an } \mathcal{F}_{T} \text {-measurable random variable such that } \mathbb{E}\left[|\xi|^{2}\right]<+\infty\right\}, \\
& S^{2}(0, T ; \mathbb{R})=\left\{\varphi \mid \varphi: \Omega \times[0, T] \rightarrow \mathbb{R} \text { is a predictable process such that } \mathbb{E}\left[\sup _{0 \leq t \leq T}\left|\varphi_{t}\right|^{2}\right]<+\infty\right\}, \\
& \mathcal{H}^{2}\left(0, T ; \mathbb{R}^{d}\right)=\left\{\varphi \mid \varphi: \Omega \times[0, T] \rightarrow \mathbb{R}^{d} \text { is a predictable process such that } \mathbb{E} \int_{0}^{T}\left|\varphi_{t}\right|^{2} \mathrm{~d} t<+\infty\right\} .
\end{aligned}
$$

We consider the following one barrier reflected BSDE with data $(f, \xi, S)$ :

$$
\left\{\begin{aligned}
Y_{t} & =\xi+\int_{t}^{T} f\left(s, Y_{s}, Z_{s}\right) \mathrm{d} s+K_{T}-K_{t}-\int_{t}^{T} Z_{s} \mathrm{~d} B_{s} \\
Y_{t} & \geq S_{t}, \quad t \in[0, T] \\
K_{0} & =0, \quad \int_{0}^{T}\left(Y_{r}-S_{r}\right) d K_{r}=0
\end{aligned}\right.
$$


where $\left\{K_{t}\right\}$ is an adapted, continuous and increasing process, $f: \Omega \times[0, T] \times \mathbb{R} \times \mathbb{R}^{d} \rightarrow \mathbb{R}$ and we make the following assumptions:

$(H 2.1) f(\cdot, 0,0) \in \mathcal{H}^{2}(0, T ; \mathbb{R})$,

(H2.2) There exists some constant $L>0$ such that for all $y, y^{\prime} \in \mathbb{R}$ and $z, z^{\prime} \in \mathbb{R}^{d}$,

$$
\left|f(t, y, z)-f\left(t, y^{\prime}, z^{\prime}\right)\right| \leq L\left(\left|y-y^{\prime}\right|+\left|z-z^{\prime}\right|\right),
$$

(H2.3) $\left\{S_{t}\right\}_{t \in[0, T]}$ is a continuous process such that $\left\{S_{t}\right\}_{0 \leq t \leq T} \in S^{2}(0, T ; \mathbb{R})$.

The following the existence and uniqueness theorem for solutions of equation (2.1) was established in [5].

Lemma 2.1. Under the assumptions $(H 2.1)-(H 2.3)$, if $\xi \in L^{2}\left(\Omega, \mathcal{F}_{T}, \mathbb{P} ; \mathbb{R}\right)$ and $S_{T} \leq \xi$, then equation (2.1) has a unique solution $(Y, Z, K)$.

We refer to $[5,12]$ for the following two estimates.

Lemma 2.2. Let the assumptions (H2.1)-(H2.3) hold and let $(Y, Z, K)$ be the solution of the reflected $B S D E$ (2.1) with data $(\xi, g, S)$. Then there exists a positive constant $C$ such that

$$
\mathbb{E}\left[\sup _{t \leq s \leq T} Y_{s}^{2}+\int_{t}^{T}\left|Z_{s}\right|^{2}+\left|K_{T}-K_{t}\right|^{2} \mid \mathcal{F}_{t}\right] \leq C \mathbb{E}\left[\xi^{2}+\left(\int_{t}^{T} g(s, 0,0) \mathrm{d} s\right)^{2}+\sup _{t \leq s \leq T} S_{s}^{2} \mid \mathcal{F}_{t}\right] .
$$

Lemma 2.3. We suppose that $(\xi, g, S)$ and $\left(\xi^{\prime}, g^{\prime}, S^{\prime}\right)$ satisfy the assumptions $(H 2.1)-(H 2.3)$. Let $(Y, Z, K)$ and $\left(Y^{\prime}, Z^{\prime}, K^{\prime}\right)$ be the solutions of the reflected BSDEs (2.1) with data $(\xi, g, S)$ and $\left(\xi^{\prime}, g^{\prime}, S^{\prime}\right)$, respectively. We let

$$
\begin{aligned}
& \Delta \xi=\xi-\xi^{\prime}, \quad \Delta g=g-g^{\prime}, \quad \Delta S=S-S^{\prime}, \\
& \Delta Y=Y-Y^{\prime}, \quad \Delta Z=Z-Z^{\prime}, \quad \Delta K=K-K^{\prime} .
\end{aligned}
$$

Then there exists a constant $C$ such that

$$
\begin{aligned}
& \mathbb{E}\left[\sup _{t \leq s \leq T}\left|\Delta Y_{s}\right|^{2}+\int_{t}^{T}\left|\Delta Z_{s}\right|^{2} \mathrm{~d} s+\left|\Delta K_{T}-\Delta K_{t}\right|^{2} \mid \mathcal{F}_{t}\right] \\
\leq & C \mathbb{E}\left[|\Delta \xi|^{2}+\left(\int_{t}^{T}\left|\Delta g\left(s, Y_{s}, Z_{s}\right)\right| \mathrm{d} s\right)^{2} \mid \mathcal{F}_{t}\right]+C\left(\mathbb{E}\left[\sup _{t \leq s \leq T}\left|\Delta S_{s}\right|^{2} \mid \mathcal{F}_{t}\right]\right)^{1 / 2} \Psi_{t, T}^{1 / 2},
\end{aligned}
$$

where

$$
\Psi_{t, T}=\mathbb{E}\left[|\xi|^{2}+\left(\int_{t}^{T}|g(s, 0,0)| \mathrm{d} s\right)^{2}+\sup _{t \leq s \leq T}\left|S_{s}\right|^{2}+\left|\xi^{\prime}\right|^{2}+\left(\int_{t}^{T}\left|g^{\prime}(s, 0,0)\right| \mathrm{d} s\right)^{2}+\sup _{t \leq s \leq T}\left|S_{s}^{\prime}\right|^{2} \mid \mathcal{F}_{t}\right] .
$$

We also need the following lemma. For its proof, the interested reader can refer to $[5,8]$ for more details.

Lemma 2.4. Let us denote by $\left(Y^{1}, Z^{1}, K^{1}\right)$ and $\left(Y^{2}, Z^{2}, K^{2}\right)$ the solutions of BSDEs with data $\left(f^{1}, \xi^{1}, S^{1}\right)$ and $\left(f^{2}, \xi^{2}, S^{2}\right)$, respectively. If $\xi^{1}, \xi^{2} \in L^{2}\left(\Omega, \mathcal{F}_{T}, \mathbb{P} ; \mathbb{R}\right), S^{1}$ and $S^{2}$ satisfy $(H 2.3)$, and $f^{1}$ and $f^{2}$ satisfy the assumptions (H2.1) and (H2.2), and the following holds

(i) $\xi^{1} \leq \xi^{2}, \mathbb{P}-$ a.s.,

(ii) $f^{1}\left(t, y_{t}^{2}, z_{t}^{2}\right) \leq f^{2}\left(t, y_{t}^{2}, z_{t}^{2}\right)$, dtdP - a.e.,

(iii) $S^{1} \leq S^{2}, \mathbb{P}-$ a.s.

Then, we have $Y_{t}^{1} \leq Y_{t}^{2}$, a.s., for all $t \in[0, T]$. Moreover, if

(iv) $f^{1}(t, y, z) \leq f^{2}(t, y, z),(t, y, z) \in[0, T] \times \mathbb{R} \times \mathbb{R}^{d}, d t d \mathbb{P}-$ a.e.,

(v) $S^{1}=S^{2}, \mathbb{P}-$ a.s.

Then, $K_{t}^{1} \geq K_{t}^{2}, \mathbb{P}-$ a.s., for all $t \in[0, T]$, and $\left\{K_{t}^{1}-K_{t}^{2}\right\}_{t \in[0, T]}$ is a increasing process. 


\section{Nonzero-Sum STOCHASTIC DifFERENTIAL GAMES With REFLECTION AND ASSOCIATED DYNAMIC PROGRAMMING PRINCIPLE}

In what follows, we assume that $U$ and $V$ are two compact metric spaces. The space $U$ is considered as the control state space of the first player, and $V$ as that of the second one. We denote the associated sets of admissible controls by $\mathcal{U}$ and $\mathcal{V}$, respectively. The set $\mathcal{U}$ is formed by all $U$-valued $\mathbb{F}$-progressively measurable processes, and $\mathcal{V}$ is the set of all $V$-valued $\mathbb{F}$-progressively measurable processes.

For given admissible controls $u(\cdot) \in \mathcal{U}$ and $v(\cdot) \in \mathcal{V}$, let us consider the following control system: for $t \in[0, T]$,

$$
\left\{\begin{aligned}
d X_{s}^{t, x ; u, v} & =b\left(s, X_{s}^{t, x ; u, v}, u_{s}, v_{s}\right) \mathrm{d} s+\sigma\left(s, X_{s}^{t, x ; u, v}, u_{s}, v_{s}\right) \mathrm{d} B_{s}, \quad s \in[t, T], \\
X_{t}^{t, x ; u, v} & =x \in \mathbb{R}^{n}
\end{aligned}\right.
$$

where

$$
b:[0, T] \times \mathbb{R}^{n} \times U \times V \rightarrow \mathbb{R}^{n}, \quad \sigma:[0, T] \times \mathbb{R}^{n} \times U \times V \rightarrow \mathbb{R}^{n \times d} .
$$

We make the following assumptions:

$(H 3.1)$ For all $x \in \mathbb{R}^{n}, b(\cdot, x, \cdot, \cdot)$ and $\sigma(\cdot, x, \cdot, \cdot)$ are continuous in $(t, u, v)$.

(H3.2) There exists a positive constant $L$ such that, for all $t \in[0, T], x, x^{\prime} \in \mathbb{R}^{n}, u \in U, v \in V$,

$$
\left|b(t, x, u, v)-b\left(t, x^{\prime}, u, v\right)\right|+\left|\sigma(t, x, u, v)-\sigma\left(t, x^{\prime}, u, v\right)\right| \leq L\left|x-x^{\prime}\right| .
$$

Under the above assumptions, for any $u(\cdot) \in \mathcal{U}$ and $v(\cdot) \in \mathcal{V}$, the control system (3.1) has a unique strong solution $\left\{X_{s}^{t, x ; u, v}, 0 \leq t \leq s \leq T\right\}$, and we also have the following standard estimates for solutions.

Lemma 3.1. For all $p \geq 2$, there exists a positive constant $C_{p}$ such that, for all $t \in[0, T], x, x^{\prime} \in \mathbb{R}^{n}, u(\cdot) \in \mathcal{U}$ and $v(\cdot) \in \mathcal{V}$,

$$
\begin{aligned}
& \mathbb{E}\left[\sup _{t \leq s \leq T}\left|X_{s}^{t, x ; u, v}\right|^{p} \mid \mathcal{F}_{t}\right] \leq C_{p}\left(1+|x|^{p}\right), \quad \mathbb{P}-\text { a.s. }, \\
& \mathbb{E}\left[\sup _{t \leq s \leq T}\left|X_{s}^{t, x ; u, v}-X_{s}^{t, x^{\prime} ; u, v}\right|^{p} \mid \mathcal{F}_{t}\right] \leq C_{p}\left|x-x^{\prime}\right|^{p}, \quad \mathbb{P}-\text { a.s. },
\end{aligned}
$$

where the constant $C_{p}$ only depends on $p$, the Lipschitz constant and the linear growth of $b$ and $\sigma$.

For given admissible controls $u(\cdot) \in \mathcal{U}$ and $v(\cdot) \in \mathcal{V}$, let us consider the following doubly controlled RBSDEs: for $j=1,2$,

$$
\left\{\begin{aligned}
{ }^{j} Y_{s}^{t, x ; u, v}= & \Phi_{j}\left(X_{T}^{t, x ; u, v}\right)+\int_{s}^{T} f_{j}\left(r, X_{r}^{t, x ; u, v},{ }^{j} Y_{r}^{t, x ; u, v},{ }^{j} Z_{r}^{t, x ; u, v}, u_{r}, v_{r}\right) \mathrm{d} r \\
& \quad+{ }^{j} K_{T}^{t, x ; u, v}-{ }^{j} K_{s}^{t, x ; u, v}-\int_{s}^{T}{ }^{j} Z_{r}^{t, x ; u, v} \mathrm{~d} B_{r}, \quad s \in[t, T], \\
{ }^{j} Y_{s}^{t, x ; u, v} \geq & h_{j}\left(s, X_{s}^{t, x ; u, v}\right), s \in[t, T], \\
{ }^{j} K_{t}^{t, x ; u, v}= & 0, \quad \int_{t}^{T}\left({ }^{j} Y_{r}^{t, x ; u, v}-h_{j}\left(r, X_{r}^{t, x ; u, v}\right)\right) d{ }^{j} K_{r}^{t, x ; u, v}=0,
\end{aligned}\right.
$$

where $X^{t, x ; u, v}$ is introduced in equation (3.1) and

$$
\begin{gathered}
\Phi_{j}=\Phi_{j}(x): \mathbb{R}^{n} \rightarrow \mathbb{R}, \quad h_{j}=h_{j}(t, x):[0, T] \times \mathbb{R}^{n} \rightarrow \mathbb{R} \\
f_{j}=f_{j}(t, x, y, z, u, v):[0, T] \times \mathbb{R}^{n} \times \mathbb{R} \times \mathbb{R}^{d} \times U \times V \rightarrow \mathbb{R} .
\end{gathered}
$$


We make the following assumptions:

(H3.3) There exists a positive constant $L$ such that, for all $t \in[0, T], x, x^{\prime} \in \mathbb{R}^{n}, y, y^{\prime} \in \mathbb{R}, z, z^{\prime} \in \mathbb{R}^{d}, u \in U$ and $v \in V, \Phi_{j}(x) \geq h_{j}(T, x)$ and

$$
\begin{aligned}
& \left|f_{j}(t, x, y, z, u, v)-f_{j}\left(t, x^{\prime}, y^{\prime}, z^{\prime}, u, v\right)\right|+\left|\Phi_{j}(x)-\Phi_{j}\left(x^{\prime}\right)\right|+\left|h_{j}(t, x)-h_{j}\left(t, x^{\prime}\right)\right| \\
& \leq L\left(\left|x-x^{\prime}\right|+\left|y-y^{\prime}\right|+\left|z-z^{\prime}\right|\right) .
\end{aligned}
$$

(H3.4) For all $(x, y, z) \in \mathbb{R}^{n} \times \mathbb{R} \times \mathbb{R}^{d}, f_{j}(\cdot, x, y, z, \cdot, \cdot)$ is continuous in $(t, u, v)$, and there exist positive constants $C$ and $\alpha \geq \frac{1}{2}$ such that, for all $t, s \in[0, T], x \in \mathbb{R}^{n}$,

$$
\left|h_{j}(t, x)-h_{j}(s, x)\right| \leq C|t-s|^{\alpha} .
$$

Under the assumption (H3.3), from [5] we know that equation (3.2) admits a unique solution. For given control processes $u(\cdot) \in U$ and $v(\cdot) \in V$, let us introduce now the associated cost functional for player $j, j=1,2$,

$$
J_{j}(t, x ; u, v):=\left.{ }^{j} Y_{s}^{t, x ; u, v}\right|_{s=t}, \quad(t, x) \in[0, T] \times \mathbb{R}^{n} .
$$

From Buckdahn and $\mathrm{Li}[4]$ we have the following estimates for solutions.

Proposition 3.2. Under the assumption (H3.1)-(H3.3), there exists a positive constant $C$ such that, for all $t \in[0, T], u(\cdot) \in \mathcal{U}$ and $v(\cdot) \in \mathcal{V}, x, x^{\prime} \in \mathbb{R}^{n}$,

$$
\begin{aligned}
& \left|{ }^{j} Y_{t}^{t, x ; u, v}\right| \leq C(1+|x|), \quad \mathbb{P}-\text { a.s. } \\
& \left|{ }^{j} Y_{t}^{t, x ; u, v}-{ }^{j} Y_{t}^{t, x^{\prime} ; u, v}\right| \leq C\left|x-x^{\prime}\right|, \quad \mathbb{P}-\text { a.s. }
\end{aligned}
$$

We now recall the definition of admissible controls and NAD strategies, which was introduced in [9].

Definition 3.3. The space $\mathcal{U}_{t, T}$ (resp., $\mathcal{V}_{t, T}$ ) of admissible controls for 1 th player (resp., 2nd) on the interval $[t, T]$ is defined as the space of all processes $\left\{u_{r}\right\}_{r \in[t, T]}$ (resp., $\left\{v_{r}\right\}_{r \in[t, T]}$ ), which are $\mathbb{F}$-progressively measurable and take values in $U$ (resp., $V$ ).

Definition 3.4. A nonanticipative strategy with delay (NAD strategy) for 1th player is a measurable mapping $\alpha: \mathcal{V}_{t, T} \rightarrow \mathcal{U}_{t, T}$, which satisfies the following properties:

1) $\alpha$ is a nonanticipative strategy, i.e., for every $\mathbb{F}$-stopping time $\tau: \Omega \rightarrow[t, T]$, and for $v_{1}, v_{2} \in \mathcal{V}_{t, T}$ with $v_{1}=v_{2}$ on $[[t, \tau]]$, it holds $\alpha\left(v_{1}\right)=\alpha\left(v_{2}\right)$ on $[[t, \tau]]$. (Recall that $[[t, \tau]]=\{(s, \omega) \in[t, T] \times \Omega, t \leq s \leq \tau(\omega)\}$ ).

2) $\alpha$ is a strategy with delay, i.e., for all $v \in \mathcal{V}_{t, T}$, there exists an increasing sequence of stopping times $\left\{S_{n}(v)\right\}_{n \geq 1}$ with

i) $t=S_{0}(v) \leq S_{1}(v) \leq \ldots \leq S_{n}(v) \leq \ldots \leq T$,

ii) $\bigcup_{n \geq 1}\left\{S_{n}(v)=T\right\}=\Omega, \mathbb{P}$-a.s.,

such that, for all $n \geq 1$ and $v, v^{\prime} \in \mathcal{V}_{t, T}, \Gamma \in \mathcal{F}_{t}$, it holds: if $v=v^{\prime}$ on $\left[\left[t, S_{n-1}(v)\right]\right] \cap([t, T] \times \Gamma)$, then

iii) $S_{l}(v)=S_{l}\left(v^{\prime}\right)$, on $\Gamma, 1 \leq l \leq n$,

iv) $\alpha(v)=\alpha\left(v^{\prime}\right)$, on $\left[\left[t, S_{n}(v)\right]\right] \bigcap([t, T] \times \Gamma)$.

We denote the set of all NAD strategies for 1 th player for games over the time interval $[t, T]$ by $\mathcal{A}_{t, T}$. The set of all NAD strategies $\beta: \mathcal{U}_{t, T} \rightarrow \mathcal{V}_{t, T}$ for 2 nd player for games over the time interval $[t, T]$ is defined in a symmetrical way and we denote it by $\mathcal{B}_{t, T}$.

NAD strategy allows us to put stochastic differential games under normal form. The following lemma was established in [9]. 
Lemma 3.5. If $(\alpha, \beta) \in \mathcal{A}_{t, T} \times \mathcal{B}_{t, T}$, then there exists a unique couple of admissible control $(u, v) \in \mathcal{U}_{t, T} \times \mathcal{V}_{t, T}$ such that

$$
\alpha(v)=u, \quad \beta(u)=v
$$

If $(\alpha, \beta) \in \mathcal{A}_{t, T} \times \mathcal{B}_{t, T}$, then from Lemma 3.5 we have a unique couple $(u, v) \in \mathcal{U}_{t, T} \times \mathcal{V}_{t, T}$ such that $(\alpha(v), \beta(u))=$ $(u, v)$. Then let us put $J_{j}(t, x ; \alpha, \beta)=J_{j}(t, x ; u, v)$. Therefore, let us define: for all $(t, x) \in[0, T] \times \mathbb{R}^{n}$,

$$
W_{j}(t, x):=\underset{\alpha \in \mathcal{A}_{t, T}}{\operatorname{esssup}} \operatorname{essinf}_{\beta \in \mathcal{B}_{t, T}} J_{j}(t, x ; \alpha, \beta),
$$

and

$$
U_{j}(t, x):=\underset{\beta \in \mathcal{B}_{t, T}}{\operatorname{essinf}} \operatorname{esssup}{ }_{\alpha \in \mathcal{A}_{t, T}} J_{j}(t, x ; \alpha, \beta) .
$$

Under the assumptions (H3.1)-(H3.3) we see that $W_{j}(t, x)$ and $U_{j}(t, x)$ are random variables. But using the arguments in $[1,10]$, we have the following proposition.

Proposition 3.6. Under the assumptions (H3.1)-(H3.3), for all $(t, x) \in[0, T] \times \mathbb{R}^{n}$, the value functions $W_{j}(t, x)$ and $U_{j}(t, x)$ are deterministic functions.

Let us now recall the definition of stochastic backward semigroups, which was first introduced by Peng [11] to study stochastic optimal control problem. For a given initial condition $(t, x) \in[0, T] \times \mathbb{R}^{n}, 0 \leq \delta \leq T-$ $t$, for admissible control processes $u(\cdot) \in \mathcal{U}_{t, t+\delta}$ and $v(\cdot) \in \mathcal{V}_{t, t+\delta}$, and a real-valued random variable $\eta \in$ $L^{2}\left(\Omega, \mathcal{F}_{t+\delta}, \mathbb{P} ; \mathbb{R}\right)$ such that $\eta \geq h_{j}\left(t+\delta, X_{t+\delta}^{t, x ; u, v}\right)$, we define

$$
{ }^{j} G_{t, t+\delta}^{t, x ; u, v}[\eta]:={ }^{j} \bar{Y}_{t}^{t, x ; u, v},
$$

where $\left({ }^{j} \bar{Y}^{t, x ; u, v},{ }^{j}{ }^{t, x ; u, v},{ }^{j} \bar{K}^{t, x ; u, v}\right)$ is the unique solution of the following reflected BSDE over the time interval $[t, t+\delta]:$

$$
\left\{\begin{aligned}
{ }^{j} \bar{Y}_{s}^{t, x ; u, v}=\eta+ & \int_{s}^{t+\delta} f_{j}\left(r, X_{r}^{t, x ; u, v},{ }^{j} \bar{Y}_{r}^{t, x ; u, v},{ }^{j} \bar{Z}_{r}^{t, x ; u, v}, u_{r}, v_{r}\right) \mathrm{d} r \\
& +{ }^{j} \bar{K}_{t+\delta}^{t, x ; u, v}-{ }^{j} \bar{K}_{s}^{t, x ; u, v}-\int_{s}^{t+\delta}{ }^{j} \bar{Z}_{r}^{t, x ; u, v} \mathrm{~d} B_{r}, \quad s \in[t, t+\delta], \\
j \bar{Y}_{s}^{t, x ; u, v} \geq & h_{j}\left(s, X_{s}^{t, x ; u, v}\right), \quad s \in[t, t+\delta], \\
{ }^{j} \bar{K}_{t}^{t, x ; u, v}= & 0, \quad \int_{t}^{t+\delta}\left({ }^{j} \bar{Y}_{r}^{t, x ; u, v}-h_{j}\left(r, X_{r}^{t, x ; u, v}\right)\right) d^{j} \bar{K}_{r}^{t, x ; u, v}=0
\end{aligned}\right.
$$

and $X^{t, x ; u, v}$ is the unique solution of equation (3.1).

For $(t, x) \in[0, T] \times \mathbb{R}^{n},(u, v) \in \mathcal{U}_{t, T} \times \mathcal{V}_{t, T}, 0 \leq \delta \leq T-t, j=1,2$, we have

$$
\begin{aligned}
J_{j}(t, x ; u, v) & ={ }^{j} G_{t, T}^{t, x ; u, v}\left[\Phi_{j}\left(X_{T}^{t, x ; u, v}\right)\right]={ }^{j} G_{t, t+\delta}^{t, x ; u, v}\left[{ }^{j} Y_{t+\delta}^{t, x ; u, v}\right] \\
& ={ }^{j} G_{t, t+\delta}^{t, x ; u, v}\left[J_{j}\left(t+\delta, X_{t+\delta}^{t, x ; u, v}, u, v\right)\right] .
\end{aligned}
$$

Remark 3.7. We consider a special case of $f_{j}$. If $f_{j}$ is independent of $(y, z)$, then we have

$$
J_{j}(t, x ; u, v)={ }^{j} G_{t, t+\delta}^{t, x ; u, v}[\eta]=\mathbb{E}\left[\eta+\int_{t}^{t+\delta} f_{j}\left(r, X_{r}^{t, x ; u, v}, u_{r}, v_{r}\right) \mathrm{d} r+{ }^{j} \bar{K}_{t+\delta}^{t, x ; u, v} \mid \mathcal{F}_{t}\right] .
$$


Proposition 3.8. Under the assumptions (H3.1)-(H3.3) we have the following dynamic programming principle: for all $0<\delta \leq T-t, x \in \mathbb{R}^{n}$,

$$
W_{j}(t, x)=\operatorname{esssup}_{\alpha \in \mathcal{A}_{t, t+\delta}} \operatorname{essinf}_{\beta \in \mathcal{B}_{t, t+\delta}}^{j} G_{t, t+\delta}^{t, x ; \alpha, \beta}\left[W_{j}\left(t+\delta, X_{t+\delta}^{t, x ; \alpha, \beta}\right)\right],
$$

and

$$
U_{j}(t, x)=\operatorname{essinf}_{\beta \in \mathcal{B}_{t, t+\delta}} \operatorname{esssup}_{\alpha \in \mathcal{A}_{t, t+\delta}}{ }^{j} G_{t, t+\delta}^{t, x ; \alpha, \beta}\left[U_{j}\left(t+\delta, X_{t+\delta}^{t, x ; \alpha, \beta}\right)\right] .
$$

The proof of the above proposition is similar to $[1,10]$, we omit the proof here.

Proposition 3.9. Under the assumptions (H3.1)-(H3.4), there exists a positive constant $C$ such that, for all $t, t^{\prime} \in[0, T]$ and $x, x^{\prime} \in \mathbb{R}^{n}$, we have

(i) $W_{j}(t, x)$ is $\frac{1}{2}$-Hölder continuous in $t$ :

$$
\left|W_{j}(t, x)-W_{j}\left(t^{\prime}, x\right)\right| \leq C(1+|x|)\left|t-t^{\prime}\right|^{\frac{1}{2}}
$$

(ii) $\left|W_{j}(t, x)-W_{j}\left(t, x^{\prime}\right)\right| \leq C\left|x-x^{\prime}\right|$.

The same properties hold true for the function $U_{j}$.

By means of the standard arguments and Proposition 3.8 we can easily get the above proposition. The proof of the above proposition is omitted here.

\section{Probabilistic interpretation of systems of IsaAcs Equations With obstacle}

The objective of this section is to give a probabilistic interpretation of systems of Isaacs equations with obstacle, and show that $W_{j}$ and $U_{j}$ introduced in Section 3, are the viscosity solutions of the following Isaacs equations with obstacle, for $(t, x) \in[0, T) \times \mathbb{R}^{n}$,

$$
\left\{\begin{array}{r}
\min \left\{W_{j}(t, x)-h_{j}(t, x),-\frac{\partial}{\partial t} W_{j}(t, x)-H_{j}^{-}\left(t, x, W_{j}(t, x), D W_{j}(t, x), D^{2} W_{j}(t, x)\right)\right\}=0 \\
W_{j}(T, x)=\Phi_{j}(x),
\end{array}\right.
$$

and

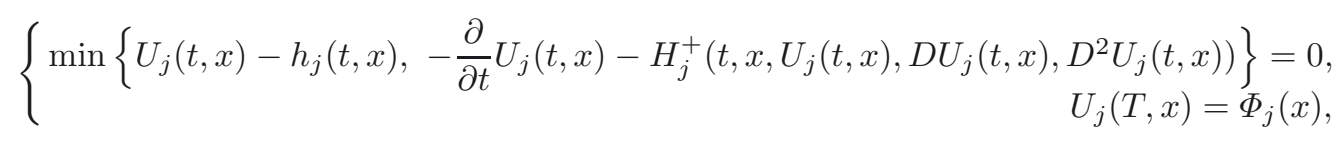

respectively, where

$$
\begin{aligned}
H_{j}(t, x, y, p, A, u, v)= & \frac{1}{2} \operatorname{tr}\left(\sigma \sigma^{T}(t, x, u, v) A\right)+p^{T} b(t, x, u, v) \\
& +f_{j}\left(t, x, y, p^{T} \sigma(t, x, u, v), u, v\right),
\end{aligned}
$$

$(t, x, y, p, u, v) \in[0, T] \times \mathbb{R}^{n} \times \mathbb{R} \times \mathbb{R}^{n} \times U \times V$ and $A \in \mathbb{S}^{n}\left(\mathbb{S}^{n}\right.$ denotes all the $n \times n$ symmetric matrices),

$$
H_{j}^{-}(t, x, y, p, A)=\sup _{u \in U} \inf _{v \in V} H_{j}(t, x, y, p, A, u, v)
$$

and

$$
H_{j}^{+}(t, x, y, p, A)=\inf _{v \in V} \sup _{u \in U} H_{j}(t, x, y, p, A, u, v) .
$$

We denote by $C_{l, b}^{3}\left([0, T] \times \mathbb{R}^{n}\right)$ the set of real-valued functions which are continuously differentiable up to the third order and whose derivatives of order from 1 to 3 are bounded. Let us recall the definition of a viscosity solution of (4.1). The definition of a viscosity solution of (4.2) can be defined in a similar way. 
Definition 4.1. For fixed $j=1,2$, let $w_{j} \in C\left([0, T] \times \mathbb{R}^{n} ; \mathbb{R}\right)$ be a function. It is called

(i) a viscosity subsolution of (4.1) if

$$
w_{j}(T, x) \leq \Phi_{j}(x), \text { for all } x \in \mathbb{R}^{n},
$$

and if for all functions $\varphi \in C_{l, b}^{3}\left([0, T] \times \mathbb{R}^{n}\right)$, and $(t, x) \in[0, T) \times \mathbb{R}^{n}$ such that $w_{j}-\varphi$ attains a local maximum at $(t, x)$,

$$
\min \left\{w_{j}(t, x)-h_{j}(t, x),-\frac{\partial}{\partial t} \varphi(t, x)-H_{j}^{-}\left(t, x, w_{j}(t, x), D \varphi(t, x), D^{2} \varphi(t, x)\right)\right\} \leq 0,
$$

(ii) a viscosity supersolution of (4.1) if

$$
w_{j}(T, x) \geq \Phi_{j}(x), \text { for all } x \in \mathbb{R}^{n},
$$

and if for all functions $\varphi \in C_{l, b}^{3}\left([0, T] \times \mathbb{R}^{n}\right)$, and $(t, x) \in[0, T) \times \mathbb{R}^{n}$ such that $w_{j}-\varphi$ attains a local minimum at $(t, x)$,

$$
\min \left\{w_{j}(t, x)-h_{j}(t, x),-\frac{\partial}{\partial t} \varphi(t, x)-H_{j}^{-}\left(t, x, w_{j}(t, x), D \varphi(t, x), D^{2} \varphi(t, x)\right)\right\} \geq 0,
$$

(iii) a viscosity solution of (4.1) if it is both a viscosity subsolution and a supersolution of (4.1).

We adapt the methods in Buckdahn and Li [4] and Buckdahn, Cardaliaguet and Quincampoix [1] to our framework. We can obtain the following theorem.

Theorem 4.2. Under the assumptions (H3.1)-(H3.3), the function $W_{j}$ (resp., $U_{j}$ ) is a viscosity solution of the system (4.1) (resp., (4.2)).

Let us now give a comparison theorem for the viscosity solution of (4.1) and (4.2). We first introduce the following space:

$$
\begin{aligned}
\Theta:=\left\{\varphi \in C\left([0, T] \times \mathbb{R}^{n}\right): \text { there exists a constant } A>0\right. \text { such that } \\
\left.\qquad \lim _{|x| \rightarrow \infty}|\varphi(t, x)| \exp \left\{-A\left[\log \left(\left(|x|^{2}+1\right)^{\frac{1}{2}}\right)\right]^{2}\right\}=0, \text { uniformly in } t \in[0, T]\right\} .
\end{aligned}
$$

Theorem 4.3. Under the assumptions (H3.1)-(H3.3), if an upper semicontinuous function $u_{1} \in \Theta$ is a viscosity subsolution of (4.1) (resp., (4.2)), and a lower semicontinuous function $u_{2} \in \Theta$ is a viscosity supersolution of (4.1) (resp., (4.2)), then we have the following:

$$
u_{1}(t, x) \leq u_{2}(t, x), \quad \text { for all }(t, x) \in[0, T] \times \mathbb{R}^{n} .
$$

By means of the arguments in Buckdahn and Li [4], we can give the proof of this theorem and the proof is omitted here.

Remark 4.4. By Proposition 3.9 we see that $W_{j}$ (resp., $U_{j}$ ) is a viscosity solution of linear growth. Therefore, from the above theorem we know that $W_{j}$ (resp., $U_{j}$ ) is the unique viscosity solution in $\Theta$ of the system (4.1) (resp., (4.2)).

\section{Isaacs condition:}

For all $(t, x, y, p, A, u, v) \in[0, T] \times \mathbb{R}^{n} \times \mathbb{R} \times \mathbb{R}^{n} \times \mathbb{S}^{n} \times U \times V, j=1,2$, we have

$$
\begin{aligned}
& \sup _{u \in U} \inf _{v \in V}\left\{\frac{1}{2} \operatorname{tr}\left(\sigma \sigma^{T}(t, x, u, v) A\right)+p^{T} b(t, x, u, v)+f_{j}\left(t, x, y, p^{T} \sigma(t, x, u, v), u, v\right)\right\} \\
= & \inf _{v \in V} \sup _{u \in U}\left\{\frac{1}{2} \operatorname{tr}\left(\sigma \sigma^{T}(t, x, u, v) A\right)+p^{T} b(t, x, u, v)+f_{j}\left(t, x, y, p^{T} \sigma(t, x, u, v), u, v\right)\right\} .
\end{aligned}
$$


Corollary 4.5. Let Isaacs condition (4.3) hold. Then we have, for all $(t, x) \in[0, T] \times \mathbb{R}^{n}$,

$$
\left(U_{1}(t, x), U_{2}(t, x)\right)=\left(W_{1}(t, x), W_{2}(t, x)\right) .
$$

In a symmetric way, for all $(t, x) \in[0, T] \times \mathbb{R}^{n}$, we put

$$
\bar{W}_{j}(t, x):=\underset{\beta \in \mathcal{B}_{t, T}}{\operatorname{esssup}} \underset{\alpha \in \mathcal{A}_{t, T}}{\operatorname{essinf}} J_{j}(t, x ; \alpha, \beta),
$$

and

$$
\bar{U}_{j}(t, x):=\underset{\alpha \in \mathcal{A}_{t, T}}{\operatorname{essinf}} \operatorname{esssup}_{\beta \in \mathcal{B}_{t, T}} J_{j}(t, x ; \alpha, \beta) .
$$

Using the arguments in $[1,10]$, we have the following propositions.

Proposition 4.6. Under the assumptions (H3.1)-(H3.3), for all $(t, x) \in[0, T] \times \mathbb{R}^{n}$, the value functions $\bar{W}_{j}(t, x)$ and $\bar{U}_{j}(t, x)$ are deterministic functions.

Proposition 4.7. Under the assumptions (H3.1)-(H3.3) we have the following dynamic programming principle: for all $0<\delta \leq T-t, x \in \mathbb{R}^{n}$,

$$
\bar{W}_{j}(t, x)=\operatorname{esssup}_{\beta \in \mathcal{B}_{t, t+\delta}} \operatorname{essinf}_{\alpha \in \mathcal{A}_{t, t+\delta}}{ }^{j} G_{t, t+\delta}^{t, x ; \alpha, \beta}\left[\bar{W}_{j}\left(t+\delta, X_{t+\delta}^{t, x ; \alpha, \beta}\right)\right],
$$

and

$$
\bar{U}_{j}(t, x)=\underset{\alpha \in \mathcal{A}_{t, t+\delta}}{\operatorname{essinf}} \operatorname{esssup}_{\beta \in \mathcal{B}_{t, t+\delta}}{ }^{j} G_{t, t+\delta}^{t, x ; \alpha}\left[\bar{U}_{j}\left(t+\delta, X_{t+\delta}^{t, x ; \alpha, \beta}\right)\right]
$$

\section{Isaacs condition:}

For all $(t, x, y, p, A, u, v) \in[0, T] \times \mathbb{R}^{n} \times \mathbb{R} \times \mathbb{R}^{n} \times \mathbb{S}^{n} \times U \times V, j=1,2$, we have

$$
\begin{aligned}
& \inf _{u \in U} \sup _{v \in V}\left\{\frac{1}{2} \operatorname{tr}\left(\sigma \sigma^{T}(t, x, u, v) A\right)+p^{T} b(t, x, u, v)+f_{j}\left(t, x, y, p^{T} \sigma(t, x, u, v), u, v\right)\right\} \\
= & \sup _{v \in V} \inf _{u \in U}\left\{\frac{1}{2} \operatorname{tr}\left(\sigma \sigma^{T}(t, x, u, v) A\right)+p^{T} b(t, x, u, v)+f_{j}\left(t, x, y, p^{T} \sigma(t, x, u, v), u, v\right)\right\} .
\end{aligned}
$$

By virtue of arguments in this section, we have the following proposition.

Proposition 4.8. Let Isaacs condition (4.4) hold. Then we have, for all $(t, x) \in[0, T] \times \mathbb{R}^{n}$,

$$
\left(\bar{U}_{1}(t, x), \bar{U}_{2}(t, x)\right)=\left(\bar{W}_{1}(t, x), \bar{W}_{2}(t, x)\right) .
$$

\section{NASH EQUILIBRIUM PAYOFFS}

The objective of this section is to obtain an existence of a Nash equilibrium payoff. For this, we consider two zero-sum stochastic differential games associated with $J_{1}$ and $J_{2}$, i.e., the first player wants to maximize $J_{1}$ and the second player wants to minimize $J_{1}$, while the first player wants to minimize $J_{2}$ and the second player wants to maximize $J_{2}$.

In what follows, we redefine the following notations which are different from the above sections: for $(t, x) \in$ $[0, T] \times \mathbb{R}^{n}$,

$$
W_{1}(t, x):=\underset{\alpha \in \mathcal{A}_{t, T}}{\operatorname{esssup}} \operatorname{essinf}_{\beta \in \mathcal{B}_{t, T}} J_{1}(t, x ; \alpha, \beta), \quad W_{2}(t, x):=\underset{\beta \in \mathcal{B}_{t, T}}{\operatorname{essup}} \operatorname{essinf} J_{\alpha \in \mathcal{A}_{t, T}}(t, x ; \alpha, \beta)
$$


We suppose that the following holds:

\section{Isaacs condition $\mathbf{A}$ :}

For all $(t, x, y, p, A, u, v) \in[0, T] \times \mathbb{R}^{n} \times \mathbb{R} \times \mathbb{R}^{n} \times \mathbb{S}^{n} \times U \times V$, we have

$$
\begin{aligned}
& \sup _{u \in U} \inf _{v \in V}\left\{\frac{1}{2} \operatorname{tr}\left(\sigma \sigma^{T}(t, x, u, v) A\right)+p^{T} b(t, x, u, v)+f_{1}\left(t, x, y, p^{T} \sigma(t, x, u, v), u, v\right)\right\} \\
= & \inf _{v \in V} \sup _{u \in U}\left\{\frac{1}{2} \operatorname{tr}\left(\sigma \sigma^{T}(t, x, u, v) A\right)+p^{T} b(t, x, u, v)+f_{1}\left(t, x, y, p^{T} \sigma(t, x, u, v), u, v\right)\right\},
\end{aligned}
$$

and

$$
\begin{aligned}
& \inf _{u \in U} \sup _{v \in V}\left\{\frac{1}{2} \operatorname{tr}\left(\sigma \sigma^{T}(t, x, u, v) A\right)+p^{T} b(t, x, u, v)+f_{2}\left(t, x, y, p^{T} \sigma(t, x, u, v), u, v\right)\right\} \\
= & \sup _{v \in V} \inf _{u \in U}\left\{\frac{1}{2} \operatorname{tr}\left(\sigma \sigma^{T}(t, x, u, v) A\right)+p^{T} b(t, x, u, v)+f_{2}\left(t, x, y, p^{T} \sigma(t, x, u, v), u, v\right)\right\} .
\end{aligned}
$$

Under the above condition, from the above section we see that: for $(t, x) \in[0, T] \times \mathbb{R}^{n}$,

$$
\begin{aligned}
& W_{1}(t, x)=\underset{\alpha \in \mathcal{A}_{t, T}}{\operatorname{essup}} \operatorname{essinf} J_{\beta \in \mathcal{B}_{t, T}}(t, x ; \alpha, \beta)=\underset{\beta \in \mathcal{B}_{t, T}}{\operatorname{essinf}} \operatorname{esssup} J_{\alpha \in \mathcal{A}_{t, T}}(t, x ; \alpha, \beta), \\
& W_{2}(t, x)=\underset{\alpha \in \mathcal{A}_{t, T}}{\operatorname{essinf}} \operatorname{esssup} J_{\beta \in \mathcal{B}_{t, T}}(t, x ; \alpha, \beta)=\underset{\beta \in \mathcal{B}_{t, T}}{\operatorname{esssup}} \underset{\alpha \in \mathcal{A}_{t, T}}{\operatorname{essinf}} J_{2}(t, x ; \alpha, \beta) .
\end{aligned}
$$

In order to simplify arguments, let us also assume that the coefficients $b, \sigma, f_{j}, \Phi_{j}, f_{j}$ and $h_{j}(j=1,2)$, satisfy the assumptions (H3.1)-(H3.4) and are bounded.

We recall the definition of the Nash equilibrium payoff of nonzero-sum stochastic differential games, which was introduced in Buckdahn, Cardaliaguet and Rainer [2] and Lin [9].

Definition 5.1. A couple $\left(e_{1}, e_{2}\right) \in \mathbb{R}^{2}$ is called a Nash equilibrium payoff at the point $(t, x)$ if for any $\varepsilon>0$, there exists $\left(\alpha_{\varepsilon}, \beta_{\varepsilon}\right) \in \mathcal{A}_{t, T} \times \mathcal{B}_{t, T}$ such that, for all $(\alpha, \beta) \in \mathcal{A}_{t, T} \times \mathcal{B}_{t, T}$,

$$
J_{1}\left(t, x ; \alpha_{\varepsilon}, \beta_{\varepsilon}\right) \geq J_{1}\left(t, x ; \alpha, \beta_{\varepsilon}\right)-\varepsilon, J_{2}\left(t, x ; \alpha_{\varepsilon}, \beta_{\varepsilon}\right) \geq J_{2}\left(t, x ; \alpha_{\varepsilon}, \beta\right)-\varepsilon, \mathbb{P}-\text { a.s. },
$$

and

$$
\left|\mathbb{E}\left[J_{j}\left(t, x ; \alpha_{\varepsilon}, \beta_{\varepsilon}\right)\right]-e_{j}\right| \leq \varepsilon, j=1,2 .
$$

From Lemma 3.5 it follows that the following lemma holds.

Lemma 5.2. For any $\varepsilon>0$ and $\left(\alpha_{\varepsilon}, \beta_{\varepsilon}\right) \in \mathcal{A}_{t, T} \times \mathcal{B}_{t, T}$, (5.2) holds if and only if, for all $(u, v) \in \mathcal{U}_{t, T} \times \mathcal{V}_{t, T}$,

$$
J_{1}\left(t, x ; \alpha_{\varepsilon}, \beta_{\varepsilon}\right) \geq J_{1}\left(t, x ; u, \beta_{\varepsilon}(u)\right)-\varepsilon, J_{2}\left(t, x ; \alpha_{\varepsilon}, \beta_{\varepsilon}\right) \geq J_{2}\left(t, x ; \alpha_{\varepsilon}(v), v\right)-\varepsilon, \mathbb{P}-a . s .
$$

Before giving the main results in this sections we first introduce the following lemma.

Lemma 5.3. Let $(t, x) \in[0, T] \times \mathbb{R}^{n}$ and $u \in \mathcal{U}_{t, T}$ be arbitrarily fixed. Then,

(i) for all $\delta \in[0, T-t]$ and $\varepsilon>0$, there exists an $N A D$ strategy $\alpha \in \mathcal{A}_{t, T}$ such that, for all $v \in \mathcal{V}_{t, T}$,

$$
\begin{aligned}
\alpha(v) & =u, \text { on }[t, t+\delta], \\
{ }^{2} Y_{t+\delta}^{t, x ; \alpha(v), v} & \leq W_{2}\left(t+\delta, X_{t+\delta}^{t, x ; \alpha(v), v}\right)+\varepsilon, \mathbb{P}-\text { a.s. }
\end{aligned}
$$


(ii) for all $\delta \in[0, T-t]$ and $\varepsilon>0$, there exists an $N A D$ strategy $\alpha \in \mathcal{A}_{t, T}$ such that, for all $v \in \mathcal{V}_{t, T}$,

$$
\begin{aligned}
\alpha(v) & =u, \text { on }[t, t+\delta], \\
{ }^{1} Y_{t+\delta}^{t, x ; \alpha(v), v} & \geq W_{1}\left(t+\delta, X_{t+\delta}^{t, x ; \alpha(v), v}\right)-\varepsilon, \mathbb{P}-\text { a.s. }
\end{aligned}
$$

Using arguments similar to Lin [9] we can prove this lemma. The proof is omitted here. We also need the following lemma, which can be established by standard arguments for SDEs.

Lemma 5.4. There exists a positive constant $C$ such that, for all $(u, v),\left(u^{\prime}, v^{\prime}\right) \in \mathcal{U}_{t, T} \times \mathcal{V}_{t, T}$, and for all $\mathcal{F}_{r}$-stopping times $S: \Omega \rightarrow[t, T]$ with $X_{S}^{t, x ; u, v}=X_{S}^{t, x ; u^{\prime}, v^{\prime}}, \mathbb{P}-$ a.s., it holds, for all real $\tau \in[0, T]$,

$$
\mathbb{E}\left[\sup _{0 \leq s \leq \tau}\left|X_{(S+s) \wedge T}^{t, x ; u, v}-X_{(S+s) \wedge T}^{t, x ; u^{\prime}, v^{\prime}}\right|^{2} \mid \mathcal{F}_{t}\right] \leq C \tau, \mathbb{P}-\text { a.s. }
$$

Let us now give one of main results in this section: the characterization of Nash equilibrium payoffs for nonzero-sum stochastic differential games with reflection. We postpone its proof to Section 6 .

Theorem 5.5. Let Isaacs condition (4.3) hold and $(t, x) \in[0, T] \times \mathbb{R}^{n}$. If for all $\varepsilon>0$, there exist $u^{\varepsilon} \in \mathcal{U}_{t, T}$ and $v^{\varepsilon} \in \mathcal{V}_{t, T}$ such that for all $s \in[t, T]$ and $j=1,2$,

$$
\mathbb{P}\left({ }^{j} Y_{s}^{t, x ; u^{\varepsilon}, v^{\varepsilon}} \geq W_{j}\left(s, X_{s}^{t, x ; u^{\varepsilon}, v^{\varepsilon}}\right)-\varepsilon \mid \mathcal{F}_{t}\right) \geq 1-\varepsilon, \mathbb{P}-\text { a.s. },
$$

and

$$
\left|\mathbb{E}\left[J_{j}\left(t, x ; u^{\varepsilon}, v^{\varepsilon}\right)\right]-e_{j}\right| \leq \varepsilon,
$$

then $\left(e_{1}, e_{2}\right) \in \mathbb{R}^{2}$ is a Nash equilibrium payoff at point $(t, x)$.

Before giving the existence theorem of a Nash equilibrium payoff we first establish the following proposition, which is crucial for the proof of the existence theorem of a Nash equilibrium payoff.

Proposition 5.6. Under the assumptions of Theorem 4.2, for all $\varepsilon>0$, there exists $\left(u^{\varepsilon}, v^{\varepsilon}\right) \in \mathcal{U}_{t, T} \times \mathcal{V}_{t, T}$ independent of $\mathcal{F}_{t}$ such that, for all $t \leq s_{1} \leq s_{2} \leq T, j=1,2$,

$$
\mathbb{P}\left(W_{j}\left(s_{1}, X_{s_{1}}^{t, x ; u^{\varepsilon}, v^{\varepsilon}}\right)-\varepsilon \leq{ }^{j} G_{s_{1}, s_{2}}^{t, x ; u^{\varepsilon}, v^{\varepsilon}}\left[W_{j}\left(s_{2}, X_{s_{2}}^{t, x ; u^{\varepsilon}, v^{\varepsilon}}\right)\right] \mid \mathcal{F}_{t}\right)>1-\varepsilon .
$$

Let us first give some preliminary result. Since the proof of the following lemma is similar to that in [9], we omit here.

Lemma 5.7. For all $\varepsilon>0$, all $\delta \in[0, T-t]$ and $x \in \mathbb{R}^{n}$, there exists $\left(u^{\varepsilon}, v^{\varepsilon}\right) \in \mathcal{U}_{t, T} \times \mathcal{V}_{t, T}$ independent of $\mathcal{F}_{t}$, such that, $j=1,2$,

$$
W_{j}(t, x)-\varepsilon \leq{ }^{j} G_{t, t+\delta}^{t, x ; \varepsilon^{\varepsilon}, v^{\varepsilon}}\left[W_{j}\left(t+\delta, X_{t+\delta}^{t, x ; u^{\varepsilon}, v^{\varepsilon}}\right)\right], \mathbb{P}-\text { a.s. }
$$

Let us establish the following Lemma.

Lemma 5.8. Let $n \geq 1$ and let us fix some partition $t=t_{0}<t_{1}<\ldots<t_{n}=T$ of the interval $[t, T]$. Then, for all $\varepsilon>0$, there exists $\left(u^{\varepsilon}, v^{\varepsilon}\right) \in \mathcal{U}_{t, T} \times \mathcal{V}_{t, T}$ independent of $\mathcal{F}_{t}$, such that, for all $i=0, \ldots, n-1$,

$$
W_{j}\left(t_{i}, X_{t_{i}}^{t, x ; u^{\varepsilon}, v^{\varepsilon}}\right)-\varepsilon \leq{ }^{j} G_{t_{i}, t_{i+1}}^{t, x ; u^{\varepsilon}, v^{\varepsilon}}\left[W_{j}\left(t_{i+1}, X_{t_{i+1}}^{t, x ; u^{\varepsilon}, v^{\varepsilon}}\right)\right], \mathbb{P}-\text { a.s. }
$$


Proof. Let us prove this lemma by induction. By the above lemma, it is obvious for $i=0$. We now suppose that $\left(u^{\varepsilon}, v^{\varepsilon}\right)$ independent of $\mathcal{F}_{t}$, is constructed on the interval $\left[t, t_{i}\right)$ and we shall define it on $\left[t_{i}, t_{i+1}\right)$. From the above lemma it follows that, for all $y \in \mathbb{R}^{n}$, there exists $\left(u^{y}, v^{y}\right) \in \mathcal{U}_{t_{i}, T} \times \mathcal{V}_{t_{i}, T}$ independent of $\mathcal{F}_{t}$, such that,

$$
W_{j}\left(t_{i}, y\right)-\frac{\varepsilon}{2} \leq{ }^{j} G_{t_{i}, t_{i+1}}^{t_{i}, y ; u^{y}, v^{y}}\left[W_{j}\left(t_{i+1}, X_{t_{i+1}}^{t, y ; u^{y}, v^{y}}\right)\right], \mathbb{P}-\text { a.s, } j=1,2 .
$$

For arbitrarily $j=1,2$, for all $y, z \in \mathbb{R}^{n}$ and $s \in\left[t_{i}, t_{i+1}\right]$, we set

$$
y_{s}^{1}={ }^{j} G_{s, t_{i+1}}^{t_{i}, y ; u^{y}, v^{y}}\left[W_{j}\left(t_{i+1}, X_{t_{i+1}}^{t_{i}, y ; u^{y}, v^{y}}\right)\right] \text {, and } y_{s}^{2}={ }^{j} G_{s, t_{i+1}}^{t_{i}, z ; u^{y}, v^{y}}\left[W_{j}\left(t_{i+1}, X_{t_{i+1}}^{t_{i}, z ; u^{y}, v^{y}}\right)\right] .
$$

Then let us consider the following BSDEs:

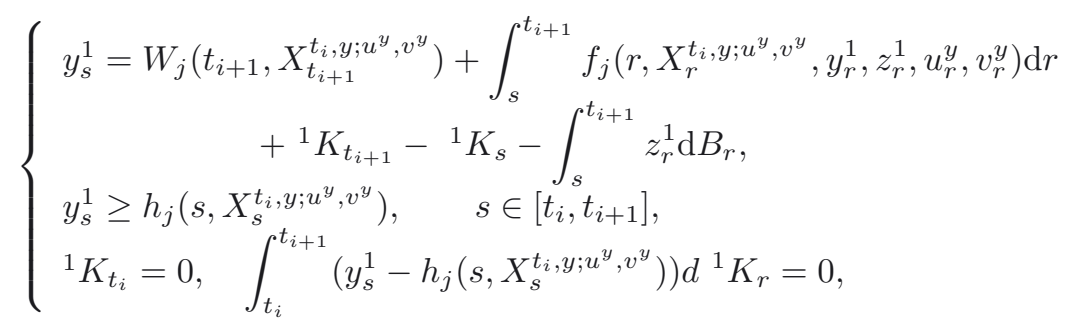

and

$$
\left\{\begin{array}{c}
y_{s}^{2}=W_{j}\left(t_{i+1}, X_{t_{i+1}}^{t_{i}, z ; u^{y}, v^{y}}\right)+\int_{s}^{t_{i+1}} f_{j}\left(r, X_{r}^{t_{i}, z ; u^{y}, v^{y}}, y_{r}^{2}, z_{r}^{2}, u_{r}^{y}, v_{r}^{y}\right) \mathrm{d} r \\
+{ }^{2} K_{t_{i+1}}-{ }^{2} K_{s}-\int_{s}^{t_{i+1}} z_{r}^{2} \mathrm{~d} B_{r}, \\
y_{s}^{2} \geq h_{j}\left(s, X_{s}^{t_{i}, z ; u^{y}, v^{y}}\right), \quad s \in\left[t_{i}, t_{i+1}\right], \\
{ }^{2} K_{t_{i}}=0, \quad \int_{t_{i}}^{t_{i+1}}\left(y_{s}^{2}-h_{j}\left(s, X_{s}^{t_{i}, z ; u^{y}, v^{y}}\right)\right) d^{2} K_{s}=0 .
\end{array}\right.
$$

From the Lemmas 2.3 and 3.1 it follows that

$$
\begin{aligned}
& \left|{ }^{j} G_{t_{i}, t_{i+1}}^{t_{i}, y, u^{y}, v^{y}}\left[W_{j}\left(t_{i+1}, X_{t_{i+1}}^{t, y ; u^{y}, v^{y}}\right)\right]-{ }^{j} G_{t_{i}, t_{i+1}}^{t_{i}, z ; u^{y}, v^{y}}\left[W_{j}\left(t_{i+1}, X_{t_{i+1}}^{t_{i}, z ; u^{y}, v^{y}}\right)\right]\right|^{2} \\
\leq & C \mathbb{E}\left[\left|W_{j}\left(t_{i+1}, X_{t_{i+1}}^{t_{i}, y ; u^{y}, v^{y}}\right)-W_{j}\left(t_{i+1}, X_{t_{i+1}}^{t_{i}, z ; u^{y}, v^{y}}\right)\right|^{2} \mid \mathcal{F}_{t_{i}}\right] \\
& +C \mathbb{E}\left[\left|\int_{t_{i}}^{t_{i+1}} f_{j}\left(r, X_{r}^{t_{i}, y ; u^{y}, v^{y}}, y_{r}^{1}, z_{r}^{1}, u_{r}^{y}, v_{r}^{y}\right) \mathrm{d} r-\int_{t_{i}}^{t_{i+1}} f_{j}\left(r, X_{r}^{t_{i}, z ; u^{y}, v^{y}}, y_{r}^{1}, z_{r}^{1}, u_{r}^{y}, v_{r}^{y}\right) \mathrm{d} r\right|^{2} \mid \mathcal{F}_{t_{i}}\right] \\
& +C \mathbb{E}\left[\sup _{t_{i} \leq s \leq t_{i+1}}\left|h_{j}\left(s, X_{s}^{t_{i}, y ; u^{y}, v^{y}}\right)-h_{j}\left(s, X_{s}^{t_{i}, z ; u^{y}, v^{y}}\right)\right|^{2} \mid \mathcal{F}_{t_{i}}\right]^{\frac{1}{2}} \\
\leq & C \mathbb{E}\left[\left|X_{t_{i+1}}^{t_{i}, y ; u^{y}, v^{y}}-X_{t_{i+1}}^{t_{i}, z ; u^{y}, v^{y}}\right|^{2} \mid \mathcal{F}_{t_{i}}\right]+C \mathbb{E}\left[\int_{t_{i}}^{t_{i+1}}\left|X_{r}^{t_{i}, y ; u^{y}, v^{y}}-X_{r}^{t_{i}, z ; u^{y}, v^{y}}\right|^{2} \mathrm{~d} r \mid \mathcal{F}_{t_{i}}\right] \\
& +C \mathbb{E}\left[\sup _{t_{i} \leq s \leq t_{i+1}}\left|X_{s}^{t_{i}, y ; u^{y}, v^{y}}-X_{s}^{t_{i}, z ; u^{y}, v^{y}}\right|^{2} \mid \mathcal{F}_{t_{i}}\right]^{\frac{1}{2}} \\
\leq & C|y-z| .
\end{aligned}
$$

Combining the above inequality, Proposition 3.9 and (5.6) we see that

$$
\begin{aligned}
W_{j}\left(t_{i}, z\right)-\varepsilon & \leq W_{j}\left(t_{i}, y\right)-\varepsilon+C|y-z|^{\frac{1}{2}} \\
& \leq{ }^{j} G_{t_{i}, t_{i+1}}^{t_{i}, u^{y}, v^{y}}\left[W_{j}\left(t_{i+1}, X_{t_{i+1}}^{t, y ; u^{y}, v^{y}}\right)\right]-\frac{\varepsilon}{2}+C|y-z|^{\frac{1}{2}} \\
& \leq{ }^{j} G_{t_{i}, t_{i+1}}^{t, z ; u^{y}, v^{y}}\left[W_{j}\left(t_{i+1}, X_{t_{i+1}}^{t, z ; u^{y}, v^{y}}\right)\right]-\frac{\varepsilon}{2}+C|y-z|^{\frac{1}{2}} \\
& \leq{ }^{j} G_{t_{i}, t_{i+1}}^{t, z ; u^{y}, v^{y}}\left[W_{j}\left(t_{i+1}, X_{t_{i+1}}^{t, z ; u^{y}, v^{y}}\right)\right], \mathbb{P}-\text { a.s. },
\end{aligned}
$$

for $C|y-z|^{\frac{1}{2}} \leq \frac{\varepsilon}{2}$. 
Let $\left\{O_{i}\right\}_{i \geq 1} \subset \mathcal{B}\left(\mathbb{R}^{n}\right)$ be a partition of $\mathbb{R}^{n}$ with $\operatorname{diam}\left(O_{i}\right)<\frac{\varepsilon}{2 C}$ and let $y_{l} \in O_{l}$. Then, for $z \in O_{l}$,

$$
W_{j}\left(t_{i}, z\right)-\varepsilon \leq{ }^{j} G_{t_{i}, t_{i+1}}^{t, z u^{y_{l}}, v^{y_{l}}}\left[W_{j}\left(t_{i+1}, X_{t_{i+1}}^{t, z ; u^{y_{l}}, v^{y_{l}}}\right)\right], \mathbb{P}-\text { a.s. }
$$

Let us put

$$
u^{\varepsilon}=\sum_{l \geq 1} 1_{O_{l}}\left(X^{t, x ; u^{\varepsilon}, v^{\varepsilon}}\right) u^{y_{l}}, v^{\varepsilon}=\sum_{l \geq 1} 1_{O_{l}}\left(X^{t, x ; u^{\varepsilon}, v^{\varepsilon}}\right) v^{y_{l}} .
$$

Then

$$
\begin{aligned}
& { }^{j} G_{t_{i}, t_{i+1}}^{t, x ; u^{\varepsilon}, v^{\varepsilon}}\left[W_{j}\left(t_{i+1}, X_{t_{i+1}}^{t, x ; u^{\varepsilon}, v^{\varepsilon}}\right)\right] \\
& ={ }^{j} G_{t_{i}, t_{i+1}}^{t_{i}, X_{i}^{t, x ; u^{\varepsilon}, v^{\varepsilon}} ; u^{\varepsilon}, v^{\varepsilon}}\left[\sum_{l \geq 1} W_{j}\left(t_{i+1}, X_{t_{i+1}}^{t_{i}, X_{t_{i}}^{t, x ; u^{\varepsilon}, v^{\varepsilon}} ; u^{\varepsilon}, v^{\varepsilon}}\right) 1_{O_{l}}\left(X_{t_{i}}^{t, x ; u^{\varepsilon}, v^{\varepsilon}}\right)\right] \\
& ={ }^{j} G_{t_{i}, t_{i+1}}^{t_{i}, X_{i}^{t, x ; u^{\varepsilon}, v^{\varepsilon}} ; u^{\varepsilon}, v^{\varepsilon}}\left[\sum_{l \geq 1} W_{j}\left(t_{i+1}, X_{t_{i+1}}^{t_{i}, X_{t_{i}}^{t, x ; u^{\varepsilon}, v^{\varepsilon}} ; u^{y_{l}}, v^{y_{l}}}\right) 1_{O_{l}}\left(X_{t_{i}}^{t, x ; u^{\varepsilon}, v^{\varepsilon}}\right)\right] \\
& =\sum_{l \geq 1}{ }^{j} G_{t_{i}, t_{i+1}}^{t_{i}, X_{i}^{t, x ; u^{\varepsilon}, v^{\varepsilon}} ; u^{y_{l}}, v^{y_{l}} l}\left[W_{j}\left(t_{i+1}, X_{t_{i+1}}^{t_{i}, X_{t_{i}}^{t, x ; u^{\varepsilon}, v^{\varepsilon}} ; u^{y_{l}}, v^{y_{l}}}\right)\right] 1_{O_{l}}\left(X_{t_{i}}^{t, x ; u^{\varepsilon}, v^{\varepsilon}}\right) \text {, }
\end{aligned}
$$

which together with (5.7) yields

$$
\begin{aligned}
& { }^{j} G_{t_{i}, t_{i+1}}^{t, x ; u^{\varepsilon}, v^{\varepsilon}}\left[W_{j}\left(t_{i+1}, X_{t_{i+1}}^{t, x ; u^{\varepsilon}, v^{\varepsilon}}\right)\right] \geq \sum_{l \geq 1}\left[W_{j}\left(t_{i}, X_{t_{i}}^{t, x ; u^{y_{l}}, v^{y_{l}}}\right)-\varepsilon\right] 1_{O_{l}}\left(X_{t_{i}}^{t, x ; u^{\varepsilon}, v^{\varepsilon}}\right) \\
= & \sum_{l \geq 1} W_{j}\left(t_{i}, X_{t_{i}}^{t, x ; u^{y_{l}}, v^{y_{l}}}\right) 1_{O_{l}}\left(X_{t_{i}}^{t, x ; u^{\varepsilon}, v^{\varepsilon}}\right)-\varepsilon=W_{j}\left(t_{i}, X_{t_{i}}^{t, x ; u^{\varepsilon}, v^{\varepsilon}}\right)-\varepsilon,
\end{aligned}
$$

from which we get the desired result.

Let us come to the proof of Proposition 5.6.

Proof. Let $t=t_{0}<t_{1}<\ldots<t_{n}=T$ be a partition of $[t, T]$, and $\tau=\sup \left(t_{i+1}-t_{i}\right)$. By Proposition 3.9 and Lemma 5.4 we see that, for all $j=1,2,0 \leq k \leq n, s \in\left[t_{k}, t_{k+1}\right)$ and $(u, v) \in \mathcal{U}_{t, T} \times \mathcal{V}_{t, T}$,

$$
\begin{aligned}
& \mathbb{E}\left[\left|W_{j}\left(t_{k}, X_{t_{k}}^{t, x ; u, v}\right)-W_{j}\left(s, X_{s}^{t, x ; u, v}\right)\right|^{2}\right] \\
\leq & 2 \mathbb{E}\left[\left|W_{j}\left(t_{k}, X_{t_{k}}^{t, x ; u, v}\right)-W_{j}\left(s, X_{t_{k}}^{t, x ; u, v}\right)\right|^{2}\right] \\
& +2 \mathbb{E}\left[\left|W_{j}\left(s, X_{t_{k}}^{t, x ; u, v}\right)-W_{j}\left(s, X_{s}^{t, x ; u, v}\right)\right|^{2}\right] \\
\leq & C\left|s-t_{k}\right|\left(1+\mathbb{E}\left[\left|X_{t_{k}}^{t, x ; u, v}\right|^{2}\right]\right)+C \mathbb{E}\left[\left|X_{t_{k}}^{t, x ; u, v}-X_{s}^{t, x ; u, v}\right|^{2}\right] \\
\leq & C \tau .
\end{aligned}
$$

Here and after $C$ represents a generic constant which may be different at different places.

We let $\left(u^{\varepsilon}, v^{\varepsilon}\right) \in \mathcal{U}_{t, T} \times \mathcal{V}_{t, T}$ be defined as in Lemma 5.8 for $\varepsilon=\varepsilon_{0}$, where $\varepsilon_{0}>0$ will be specified later. Then, for all $i, 0 \leq i \leq n$,

$$
W_{j}\left(t_{i}, X_{t_{i}}^{t, x ; u^{\varepsilon}, v^{\varepsilon}}\right)-\varepsilon_{0} \leq{ }^{j} G_{t_{i}, t_{i+1}}^{t, x ; u^{\varepsilon}, v^{\varepsilon}}\left[W_{j}\left(t_{i+1}, X_{t_{i+1}}^{t, x ; u^{\varepsilon}, v^{\varepsilon}}\right)\right], \mathbb{P}-\text { a.s. }
$$


For $t \leq s_{1} \leq s_{2} \leq T$, let us suppose, without loss of generality, that $t_{i-1} \leq s_{1}<t_{i}$ and $t_{k}<s_{2} \leq t_{k+1}$, for some $1 \leq i<k \leq n-1$. Therefore, applying the Lemmas 2.3 and 2.4 we deduce that

$$
\begin{aligned}
& { }^{j} G_{t_{i}, t_{k+1}}^{t, x ; t^{\varepsilon}, v^{\varepsilon}}\left[W_{j}\left(t_{k+1}, X_{t_{k+1}}^{t, x ; u^{\varepsilon}, v^{\varepsilon}}\right)\right]={ }^{j} G_{t_{i}, t_{k}}^{t, x ; u^{\varepsilon}, v^{\varepsilon}}\left[{ }^{j} G_{t_{k}, t_{k+1}}^{t, x ; u^{\varepsilon}, v^{\varepsilon}}\left[W_{j}\left(t_{k+1}, X_{t_{k+1}}^{t, x ; u^{\varepsilon}, v^{\varepsilon}}\right)\right]\right] \\
& \geq{ }^{j} G_{t_{i}, t_{k}}^{t, x ; u^{\varepsilon}, v^{\varepsilon}}\left[W_{j}\left(t_{k}, X_{t_{k}}^{t, x ; u^{\varepsilon}, v^{\varepsilon}}\right)-\varepsilon_{0}\right] \\
& \geq{ }^{j} G_{t_{i}, t_{k}}^{t, x ; u^{\varepsilon}, v^{\varepsilon}}\left[W_{j}\left(t_{k}, X_{t_{k}}^{t, x ; u^{\varepsilon}, v^{\varepsilon}}\right)\right]-C \varepsilon_{0} \\
& \geq \ldots \geq{ }^{j} G_{t_{i}, t_{i+1}}^{t, x u^{\varepsilon}}\left[W_{j}\left(t_{i+1}, X_{t_{i+1}}^{t, x ; u^{\varepsilon}, v^{\varepsilon}}\right)\right]-C(k-i) \varepsilon_{0} \\
& \geq W_{j}\left(t_{i}, X_{t_{i}}^{t, x ; u^{\varepsilon}, v^{\varepsilon}}\right)-C(k-i+1) \varepsilon_{0},
\end{aligned}
$$

from which we see that

$$
\begin{aligned}
{ }^{j} G_{s_{1}, t_{k+1}}^{t, x ; u^{\varepsilon}, v^{\varepsilon}}\left[W_{j}\left(t_{k+1}, X_{t_{k+1}}^{t, x ; u^{\varepsilon}, v^{\varepsilon}}\right)\right] & ={ }^{j} G_{s_{1}, t_{i}}^{t, x ; u^{\varepsilon}, v^{\varepsilon}}\left[{ }^{j} G_{t_{i}, t_{k+1}}^{t, x ; u^{\varepsilon}, v^{\varepsilon}}\left[W_{j}\left(t_{k+1}, X_{t_{k+1}}^{t, x ; u^{\varepsilon}, v^{\varepsilon}}\right)\right]\right] \\
& \geq{ }^{j} G_{s_{1}, t_{i}}^{t, x ; u^{\varepsilon}, v^{\varepsilon}}\left[W_{j}\left(t_{i}, X_{t_{i}}^{t, x ; u^{\varepsilon}, v^{\varepsilon}}\right)-C(k-i+1) \varepsilon_{0}\right] \\
& \geq{ }^{j} G_{s_{1}, t_{i}}^{t, x ; u^{\varepsilon}, v^{\varepsilon}}\left[W_{j}\left(t_{i}, X_{t_{i}}^{t, x ; u^{\varepsilon}, v^{\varepsilon}}\right)\right]-C(k-i+2) \varepsilon_{0} \\
& \geq{ }^{j} G_{s_{1}, t_{i}}^{t, x ; u^{\varepsilon}, v^{\varepsilon}}\left[W_{j}\left(t_{i}, X_{t_{i}}^{t, x ; u^{\varepsilon}, v^{\varepsilon}}\right)\right]-\frac{\varepsilon}{2},
\end{aligned}
$$

where we put $\varepsilon_{0}=\frac{\varepsilon}{2 C n}$. We set

$$
\begin{aligned}
& I_{1}={ }^{j} G_{s_{1}, t_{k+1}}^{t, x ; u^{\varepsilon}, v^{\varepsilon}}\left[W_{j}\left(t_{k+1}, X_{t_{k+1}}^{t, x ; u^{\varepsilon}, v^{\varepsilon}}\right)\right]-{ }^{j} G_{s_{1}, t_{i}}^{t, x ; u^{\varepsilon}, v^{\varepsilon}}\left[W_{j}\left(t_{i}, X_{t_{i}}^{t, x ; u^{\varepsilon}, v^{\varepsilon}}\right)\right]+\frac{\varepsilon}{2} \geq 0, \\
& I_{2}={ }^{j} G_{s_{1}, s_{2}}^{t, x ; u^{\varepsilon}, v^{\varepsilon}}\left[W_{j}\left(s_{2}, X_{s_{2}}^{t, x ; u^{\varepsilon}, v^{\varepsilon}}\right)\right]-W_{j}\left(s_{1}, X_{s_{1}}^{t, x ; u^{\varepsilon}, v^{\varepsilon}}\right)+\frac{\varepsilon}{2} .
\end{aligned}
$$

In what follows we shall prove the following:

$$
\mathbb{E}\left[\left|I_{1}-I_{2}\right|^{2}\right] \leq C \tau .
$$

Let us put

$$
y_{s}={ }^{j} G_{s, t_{i}}^{t, x ; u^{\varepsilon}, v^{\varepsilon}}\left[W_{j}\left(t_{i}, X_{t_{i}}^{t, x ; u^{\varepsilon}, v^{\varepsilon}}\right)\right], s \in\left[s_{1}, t_{i}\right] .
$$

Then we consider the associated BSDEs:

$$
\left\{\begin{array}{l}
y_{s}=W_{j}\left(t_{i}, X_{t_{i}}^{t, x ; u^{\varepsilon}, v^{\varepsilon}}\right)+\int_{s}^{t_{i}} f_{j}\left(r, X_{r}^{t, x ; u^{\varepsilon}, v^{\varepsilon}}, y_{r}, z_{r}, u_{r}^{\varepsilon}, v_{r}^{\varepsilon}\right) \mathrm{d} r+k_{t_{i}}-k_{s}-\int_{s}^{t_{i}} z_{r} \mathrm{~d} B_{r} \\
y_{s} \geq h_{j}\left(s, X_{s}^{t, x ; u^{\varepsilon}, v^{\varepsilon}}\right), \quad s \in\left[s_{1}, t_{i}\right] \\
k_{s_{1}}=0, \quad \int_{s_{1}}^{t_{i}}\left(y_{r}-h_{j}\left(r, X_{r}^{t, x ; u^{\varepsilon}, v^{\varepsilon}}\right)\right) d k_{r}=0
\end{array}\right.
$$

and

$$
y_{s}^{\prime}=W_{j}\left(s_{1}, X_{s_{1}}^{t, x ; u^{\varepsilon}, v^{\varepsilon}}\right), s \in\left[s_{1}, t_{i}\right] .
$$

Thus, applying Lemma 2.3 we conclude

$$
\begin{aligned}
& \left|{ }^{j} G_{s_{1}, t_{i}}^{t, x ; v^{\varepsilon}}\left[W_{j}\left(t_{i}, X_{t_{i}}^{t, x ; u^{\varepsilon}, v^{\varepsilon}}\right)\right]-W_{j}\left(s_{1}, X_{s_{1}}^{t, x ; u^{\varepsilon}, v^{\varepsilon}}\right)\right|^{2} \\
\leq & C \mathbb{E}\left[\left|W_{j}\left(t_{i}, X_{t_{i}}^{t, x ; u^{\varepsilon}, v^{\varepsilon}}\right)-W_{j}\left(s_{1}, X_{s_{1}}^{t, x ; u^{\varepsilon}, v^{\varepsilon}}\right)\right|^{2} \mid \mathcal{F}_{s_{1}}\right] \\
& +C \mathbb{E}\left[\int_{s_{1}}^{t_{i}}\left|f_{j}\left(r, X_{r}^{t, x ; u^{\varepsilon}, v^{\varepsilon}}, y_{r}, z_{r}, u_{r}^{\varepsilon}, v_{r}^{\varepsilon}\right)\right|^{2} \mid \mathcal{F}_{s_{1}}\right] \\
& +C \mathbb{E}\left[\sup _{s_{1} \leq s \leq t_{i}}\left|h_{j}\left(s, X_{s}^{t, x ; u^{\varepsilon}, v^{\varepsilon}}\right)-h_{j}\left(s_{1}, X_{s_{1}}^{t, x ; u^{\varepsilon}, v^{\varepsilon}}\right)\right|^{2} \mathrm{~d} r \mid \mathcal{F}_{s_{1}}\right]^{\frac{1}{2}} \\
\leq & C \mathbb{E}\left[\left|W_{j}\left(t_{i}, X_{t_{i}}^{t, x ; u^{\varepsilon}, v^{\varepsilon}}\right)-W_{j}\left(s_{1}, X_{s_{1}}^{t, x ; u^{\varepsilon}, v^{\varepsilon}}\right)\right|^{2} \mid \mathcal{F}_{s_{1}}\right] \\
& +C\left(t_{i}-s_{1}\right)^{\alpha}+C \mathbb{E}\left[\sup _{s_{1} \leq s \leq t_{i}}\left|X_{s}^{t, x ; u^{\varepsilon}, v^{\varepsilon}}-X_{s_{1}}^{t, x ; u^{\varepsilon}, v^{\varepsilon}}\right|^{2} \mid \mathcal{F}_{s_{1}}\right]^{\frac{1}{2}}
\end{aligned}
$$


where we have used the assumptions (H3.3) and (H3.4) and the boundedness of $f_{j}$. Since $\left(u^{\varepsilon}, v^{\varepsilon}\right) \in \mathcal{U}_{t, T} \times \mathcal{V}_{t, T}$ is independent of $\mathcal{F}_{t}$ we have

$$
\begin{aligned}
& \mathbb{E}\left[\left.\right|^{j} G_{s_{1}, t_{i}}^{t, x ; u^{\varepsilon}, v^{\varepsilon}}\left[W_{j}\left(t_{i}, X_{t_{i}}^{t, x ; u^{\varepsilon}, v^{\varepsilon}}\right)\right]-\left.W_{j}\left(s_{1}, X_{s_{1}}^{t, x ; u^{\varepsilon}, v^{\varepsilon}}\right)\right|^{2} \mid \mathcal{F}_{t}\right] \\
\leq & C \mathbb{E}\left[\left|W_{j}\left(t_{i}, X_{t_{i}}^{t, x ; u^{\varepsilon}, v^{\varepsilon}}\right)-W_{j}\left(s_{1}, X_{s_{1}}^{t, x ; u^{\varepsilon}, v^{\varepsilon}}\right)\right|^{2}\right]+C\left(t_{i}-s_{1}\right) \\
& +C \mathbb{E}\left[\sup _{s_{1} \leq s \leq t_{i}}\left|X_{s}^{t, x ; u^{\varepsilon}, v^{\varepsilon}}-X_{s_{1}}^{t, x ; u^{\varepsilon}, v^{\varepsilon}}\right|^{2} \mid \mathcal{F}_{t}\right]^{\frac{1}{2}}
\end{aligned}
$$

By virtue of (5.8) we have

$$
\mathbb{E}\left[{ }^{j} G_{s_{1}, t_{i}}^{t, x ; v^{\varepsilon}}, v^{\varepsilon}\left[W_{j}\left(t_{i}, X_{t_{i}}^{t, x ; u^{\varepsilon}, v^{\varepsilon}}\right)\right]-\left.W_{j}\left(s_{1}, X_{s_{1}}^{t, x ; u^{\varepsilon}, v^{\varepsilon}}\right)\right|^{2}\right] \leq C \tau^{\frac{1}{2}} .
$$

By a similar argument

$$
\mathbb{E}\left[\left.\right|^{j} G_{s_{2}, t_{k+1}}^{t, x ; u^{\varepsilon}, v^{\varepsilon}}\left[W_{j}\left(t_{k+1}, X_{t_{k+1}}^{t, x ; u^{\varepsilon}, v^{\varepsilon}}\right)\right]-\left.W_{j}\left(s_{2}, X_{s_{2}}^{t, x ; u^{\varepsilon}, v^{\varepsilon}}\right)\right|^{2}\right] \leq C \tau^{\frac{1}{2}} .
$$

For $s \in\left[s_{1}, s_{2}\right]$ we put

$$
y_{s}^{1}={ }^{j} G_{s, t_{k+1}}^{t, x ; u^{\varepsilon}, v^{\varepsilon}}\left[W_{j}\left(t_{k+1}, X_{t_{k+1}}^{t, x ; u^{\varepsilon}, v^{\varepsilon}}\right)\right]={ }^{j} G_{s, s_{2}}^{t, x ; u^{\varepsilon}, v^{\varepsilon}}\left[{ }^{j} G_{s_{2}, t_{k+1}}^{t, x ; u^{\varepsilon}}\left[v^{\varepsilon}\left[W_{j+1}\left(t_{k+1}, X_{t_{k+1}}^{t, x ; u^{\varepsilon}, v^{\varepsilon}}\right)\right]\right],\right.
$$

and

$$
y_{s}^{2}={ }^{j} G_{s, s_{2}}^{t, x ; u^{\varepsilon}, v^{\varepsilon}}\left[W_{j}\left(s_{2}, X_{s_{2}}^{t, x ; u^{\varepsilon}, v^{\varepsilon}}\right)\right] .
$$

Let us consider the associated BSDEs:

$$
\left\{\begin{array}{c}
y_{s}^{1}={ }^{j} G_{s_{2}, t_{k+1}}^{t, x ; u^{\varepsilon}, v^{\varepsilon}}\left[W_{j}\left(t_{k+1}, X_{t_{k+1}}^{t, x ; u^{\varepsilon}, v^{\varepsilon}}\right)\right]+\int_{s}^{s_{2}} f_{j}\left(r, X_{r}^{t, x ; u^{\varepsilon}, v^{\varepsilon}}, y_{r}^{1}, z_{r}^{1}, u_{r}^{\varepsilon}, v_{r}^{\varepsilon}\right) \mathrm{d} r \\
+k_{s_{2}}^{1}-k_{s}^{1}-\int_{s}^{s_{2}} z_{r}^{1} \mathrm{~d} B_{r} \\
y_{s}^{1} \geq h_{j}\left(s, X_{s}^{t, x ; u^{\varepsilon}, v^{\varepsilon}}\right), \quad s \in\left[s_{1}, s_{2}\right] \\
k_{s_{1}}^{1}=0, \quad \int_{s_{1}}^{s_{2}}\left(y_{r}-h_{j}\left(r, X_{r}^{t, x ; u^{\varepsilon}, v^{\varepsilon}}\right)\right) d k_{r}^{1}=0
\end{array}\right.
$$

and

$$
\left\{\begin{array}{c}
y_{s}^{2}=W_{j}\left(s_{2}, X_{s_{2}}^{t, x ; u^{\varepsilon}, v^{\varepsilon}}\right)+\int_{s}^{s_{2}} f_{j}\left(r, X_{r}^{t, x ; u^{\varepsilon}, v^{\varepsilon}}, y_{r}^{2}, z_{r}^{2}, u_{r}^{\varepsilon}, v_{r}^{\varepsilon}\right) \mathrm{d} r \\
+k_{s_{2}}^{2}-k_{s}^{2}-\int_{s}^{s_{2}} z_{r}^{2} \mathrm{~d} B_{r} \\
y_{s}^{2} \geq h_{j}\left(s, X_{s}^{t, x ; u^{\varepsilon}, v^{\varepsilon}}\right), \quad s \in\left[s_{1}, s_{2}\right] \\
k_{s_{1}}^{2}=0, \quad \int_{s_{1}}^{s_{2}}\left(y_{r}-h_{j}\left(r, X_{r}^{t, x ; u^{\varepsilon}, v^{\varepsilon}}\right)\right) d k_{r}^{2}=0
\end{array}\right.
$$

From the Lemmas 2.3 and 3.1 it follows that

$$
\begin{aligned}
& \left|{ }^{j} G_{s_{1}, t_{k+1}}^{t, x ; v^{\varepsilon}, v^{\varepsilon}}\left[W_{j}\left(t_{k+1}, X_{t_{k+1}}^{t, x ; u^{\varepsilon}, v^{\varepsilon}}\right)\right]-{ }^{j} G_{s_{1}, s_{2}}^{t, x ; u^{\varepsilon}, v^{\varepsilon}}\left[W_{j}\left(s_{2}, X_{s_{2}}^{t, x ; u^{\varepsilon}, v^{\varepsilon}}\right)\right]\right|^{2} \\
& \leq C \mathbb{E}\left[\left|{ }^{j} G_{s_{2}, t_{k+1}}^{t, x ; u^{\varepsilon}}\left[v_{j}^{\varepsilon}\left[W_{k+1}, X_{t_{k+1}}^{t, x ; u^{\varepsilon}, v^{\varepsilon}}\right)\right]-W_{j}\left(s_{2}, X_{s_{2}}^{t, x ; u^{\varepsilon}, v^{\varepsilon}}\right)\right|^{2} \mid \mathcal{F}_{s_{1}}\right] .
\end{aligned}
$$

Hence, by (5.11) we see that

$$
\mathbb{E}\left[\left|{ }^{j} G_{s_{1}, t_{k+1}}^{t, x ; u^{\varepsilon}, v^{\varepsilon}}\left[W_{j}\left(t_{k+1}, X_{t_{k+1}}^{t, x ; u^{\varepsilon}, v^{\varepsilon}}\right)\right]-{ }^{j} G_{s_{1}, s_{2}}^{t, x ; u^{\varepsilon}}, v^{\varepsilon}\left[W_{j}\left(s_{2}, X_{s_{2}}^{t, x ; u^{\varepsilon}, v^{\varepsilon}}\right)\right]\right|^{2}\right] \leq C \tau^{\frac{1}{2}} .
$$


The above inequality and (5.10) yield

$$
\mathbb{E}\left[\left|I_{1}-I_{2}\right|^{2}\right] \leq C \tau^{\frac{1}{2}}
$$

Therefore,

$$
\mathbb{P}\left(I_{2} \leq-\frac{\varepsilon}{2}\right) \leq \mathbb{P}\left(\left|I_{1}-I_{2}\right| \geq \frac{\varepsilon}{2}\right) \leq \frac{4 \mathbb{E}\left[\left|I_{1}-I_{2}\right|^{2}\right]}{\varepsilon^{2}} \leq \frac{4 C \tau^{\frac{1}{2}}}{\varepsilon^{2}} \leq \varepsilon
$$

where we choose $\tau \leq\left(\frac{\varepsilon^{3}}{4 C}\right)^{2}$, and by (5.9) we have

$$
\mathbb{P}\left(W_{j}\left(s_{1}, X_{s_{1}}^{t, x ; u^{\varepsilon}, v^{\varepsilon}}\right)-\varepsilon \leq{ }^{j} G_{s_{1}, s_{2}}^{t, x ; u^{\varepsilon}, v^{\varepsilon}}\left[W_{j}\left(s_{2}, X_{s_{2}}^{t, x ; u^{\varepsilon}, v^{\varepsilon}}\right)\right]\right) \geq 1-\varepsilon .
$$

We also refer to the fact that since $\left(u^{\varepsilon}, v^{\varepsilon}\right)$ is independent of $\mathcal{F}_{t}$, the conditional probability $\mathbb{P}\left(\cdot \mid \mathcal{F}_{t}\right)$ of the event $\left\{W_{j}\left(s_{1}, X_{s_{1}}^{t, x ; u^{\varepsilon}, v^{\varepsilon}}\right)-\varepsilon \leq{ }^{j} G_{s_{1}, s_{2}}^{t, x ; u^{\varepsilon}}, v^{\varepsilon}\left[W_{j}\left(s_{2}, X_{s_{2}}^{\left.t, x ; u^{\varepsilon}, v^{\varepsilon}\right)}\right)\right\}\right.$ coincides with its probability. Indeed, also

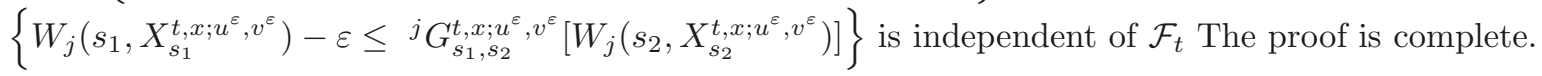

Finally, we give another main result: the existence theorem of a Nash equilibrium payoff.

Theorem 5.9. Under the Isaacs condition $A$, for all $(t, x) \in[0, T] \times \mathbb{R}^{n}$, there exists a Nash equilibrium payoff at $(t, x)$.

Proof. By Theorem 4.2 we only have to prove that, for all $\varepsilon>0$, there exists $\left(u^{\varepsilon}, v^{\varepsilon}\right) \in \mathcal{U}_{t, T} \times \mathcal{V}_{t, T}$ which satisfies (5.4) and (5.5) for $s \in[t, T], j=1,2$. For $\varepsilon>0$, let us consider $\left(u^{\varepsilon}, v^{\varepsilon}\right) \in \mathcal{U}_{t, T} \times \mathcal{V}_{t, T}$ given by Proposition 5.6, i.e., in particular, $\left(u^{\varepsilon}, v^{\varepsilon}\right)$ is independent of $\mathcal{F}_{t}$. Setting $s_{1}=t$ and $s_{2}=T$ in Proposition 5.6, we get (5.4). Since $\left(u^{\varepsilon}, v^{\varepsilon}\right)$ is independent of $\mathcal{F}_{t}, J_{j}\left(t, x ; u^{\varepsilon}, v^{\varepsilon}\right), j=1,2$, are deterministic and $\left\{\left(J_{1}\left(t, x ; u^{\varepsilon}, v^{\varepsilon}\right), J_{2}\left(t, x ; u^{\varepsilon}, v^{\varepsilon}\right)\right), \varepsilon>0\right\}$ is a bounded sequence. Therefore, we can choose an accumulation point of this sequence, as $\varepsilon \rightarrow 0$. We denote this point by $\left(e_{1}, e_{2}\right)$. From Theorem 4.2 we see that $\left(e_{1}, e_{2}\right)$ is a Nash equilibrium payoff at $(t, x)$. The proof is complete.

\section{Proof of Theorem 4.2}

We now give the Proof of Theorem 4.2.

Proof. For arbitrarily fixed $\varepsilon>0$ and some $\varepsilon_{0}>0$ ( $\varepsilon_{0}$ depends on $\varepsilon$ and will be precise later), let us assume that $\left(u^{\varepsilon_{0}}, v^{\varepsilon_{0}}\right) \in \mathcal{U}_{t, T} \times \mathcal{V}_{t, T}$ satisfies (5.4) and (5.5), i.e., for all $s \in[t, T]$ and $j=1,2$,

$$
\mathbb{P}\left({ }^{j} Y_{s}^{t, x ; u^{\varepsilon_{0}}, v^{\varepsilon_{0}}} \geq W_{j}\left(s, X_{s}^{t, x ; u^{\varepsilon_{0}}, v^{\varepsilon_{0}}}\right)-\varepsilon_{0} \mid \mathcal{F}_{t}\right) \geq 1-\varepsilon_{0}, \mathbb{P}-\text { a.s. },
$$

and

$$
\left|\mathbb{E}\left[J_{j}\left(t, x ; u^{\varepsilon_{0}}, v^{\varepsilon_{0}}\right)\right]-e_{j}\right| \leq \varepsilon_{0} .
$$

We fix some partition: $t=t_{0} \leq t_{1} \leq \ldots \leq t_{m}=T$ of $[t, T]$ and put $\tau=\sup _{i}\left|t_{i}-t_{i+1}\right|$. Let us apply Lemma 5.3 to $u^{\varepsilon_{0}}$ and $t+\delta=t_{1}, \ldots, t_{m}$, successively. Then, for $\varepsilon_{1}>0\left(\varepsilon_{1}\right.$ depends on $\varepsilon$ and is specified later $)$ we have the existence of NAD strategies $\alpha_{i} \in \mathcal{A}_{t, T}, i=1, \ldots, m$, such that, for all $v \in \mathcal{V}_{t, T}$,

$$
\begin{aligned}
\alpha_{i}(v) & =u^{\varepsilon_{0}}, \text { on }\left[t, t_{i}\right], \\
{ }^{2} Y_{t_{i}}^{t, x ; \alpha_{i}(v), v} & \leq W_{2}\left(t_{i}, X_{t_{i}}^{t, x ; \alpha_{i}(v), v}\right)+\varepsilon_{1}, \mathbb{P}-\text { a.s. }
\end{aligned}
$$


For all $v \in \mathcal{V}_{t, T}$, we set

$$
\begin{aligned}
& S^{v}=\inf \left\{s \geq t \mid \lambda\left(\left\{r \in[t, s]: v_{r} \neq v_{r}^{\varepsilon_{0}}\right\}\right)>0\right\} \\
& t^{v}=\inf \left\{t_{i} \geq S^{v} \mid i=1, \ldots, m\right\} \wedge T .
\end{aligned}
$$

Here $\lambda$ denotes the Lebesgue measure on the real line $\mathbb{R}$. We see that $S^{v}$ and $t^{v}$ are stopping times such that $S^{v} \leq t^{v} \leq S^{v}+\tau$.

We put

$$
\alpha_{\varepsilon}(v)= \begin{cases}u^{\varepsilon_{0}}, & \text { on }\left[\left[t, t^{v}\right]\right] \\ \alpha_{i}(v), & \text { on }\left(t_{i}, T\right] \times\left\{t^{v}=t_{i}\right\}, 1 \leq i \leq m .\end{cases}
$$

Then, $\alpha_{\varepsilon}$ is an NAD strategy. It follows from (6.3) that

$$
\begin{aligned}
{ }^{2} Y_{t^{v}}^{t, x ; \alpha_{\varepsilon}(v), v} & =\sum_{i=1}^{m}{ }^{2} Y_{t_{i}}^{t, x ; \alpha_{\varepsilon}(v), v} 1_{\left\{t^{v}=t_{i}\right\}} \\
& \leq \sum_{i=1}^{m} W_{2}\left(t_{i}, X_{t_{i}}^{t, x ; \alpha_{\varepsilon}(v), v}\right) 1_{\left\{t^{v}=t_{i}\right\}}+\varepsilon_{1} \\
& =W_{2}\left(t^{v}, X_{t^{v}}^{t, x ; \alpha_{\varepsilon}(v), v}\right)+\varepsilon_{1}, \mathbb{P}-\text { a.s. }
\end{aligned}
$$

Let us show that, for all $\varepsilon>0$ and $v \in \mathcal{V}_{t, T}$,

$$
J_{2}\left(t, x ; \alpha_{\varepsilon}(v), v\right) \leq J_{2}\left(t, x ; u^{\varepsilon_{0}}, v^{\varepsilon_{0}}\right)+\varepsilon, \quad \alpha_{\varepsilon}\left(v^{\varepsilon_{0}}\right)=u^{\varepsilon_{0}} .
$$

Thanks to (6.4), from Lemmas 2.3 and 2.4 we see that there exists a positive constant $C$ such that

$$
\begin{aligned}
J_{2}\left(t, x, \alpha_{\varepsilon}(v), v\right) & ={ }^{2} G_{t, t^{v}}^{t, x ; \alpha_{\varepsilon}(v), v}\left[{ }^{2} Y_{t^{v}}^{t, x, \alpha_{\varepsilon}(v), v}\right] \\
& \leq{ }^{2} G_{t, t^{v}}^{t, x ; \alpha_{\varepsilon}(v), v}\left[W_{2}\left(t^{v}, X_{t^{v}}^{t, x ; \alpha_{\varepsilon}(v), v}\right)+\varepsilon_{1}\right] \\
& \leq{ }^{2} G_{t, t^{v}}^{t, x ; \alpha_{\varepsilon}(v), v}\left[W_{2}\left(t^{v}, X_{t^{v}}^{t, x ; \alpha_{\varepsilon}(v), v}\right)\right]+C \varepsilon_{1} .
\end{aligned}
$$

Therefore, from Lemma 2.3

$$
\begin{aligned}
& \left|{ }^{2} G_{t, t^{v}}^{t, x ; \alpha_{\varepsilon}(v), v}\left[W_{2}\left(t^{v}, X_{t^{v}}^{t, x ; u^{\varepsilon_{0}}, v^{\varepsilon_{0}}}\right)\right]-{ }^{2} G_{t, t^{v}}^{t, x ; \alpha_{\varepsilon}(v), v}\left[W_{2}\left(t^{v}, X_{t^{v}}^{t, x ; \alpha_{\varepsilon}(v), v}\right)\right]\right| \\
\leq & C \mathbb{E}\left[\left|W_{2}\left(t^{v}, X_{t^{v}}^{t, x ; u^{\varepsilon_{0}}, v^{\varepsilon_{0}}}\right)-W_{2}\left(t^{v}, X_{t^{v}}^{t, x ; \alpha_{\varepsilon}(v), v}\right)\right|^{2} \mid \mathcal{F}_{t}\right]^{\frac{1}{2}} \\
\leq & C \mathbb{E}\left[\left|X_{t^{v}}^{t, x ; u^{\varepsilon_{0}}, v^{\varepsilon_{0}}}-X_{t^{v}}^{t, x ; \alpha_{\varepsilon}(v), v}\right|^{2} \mid \mathcal{F}_{t}\right]^{\frac{1}{2}} \\
\leq & C \tau^{\frac{1}{2}}, \mathbb{P}-\text { a.s. },
\end{aligned}
$$

for the last two inequalities we have used Proposition 3.9 and Lemma 5.4. Then, (6.6) yields

$$
\begin{aligned}
J_{2}\left(t, x, \alpha_{\varepsilon}(v), v\right) \leq & { }^{2} G_{t, t^{v}}^{t, x ; \alpha_{\varepsilon}(v), v}\left[W_{2}\left(t^{v}, X_{t^{v}}^{t, x ; u^{\varepsilon_{0}}, v^{\varepsilon_{0}}}\right)\right]+C \varepsilon_{1} \\
& +\left|{ }^{2} G_{t, t^{v}}^{t, x ; \alpha_{\varepsilon}(v), v}\left[W_{2}\left(t^{v}, X_{t^{v}}^{t, x ; u^{\varepsilon_{0}}, v^{\varepsilon_{0}}}\right)\right]-{ }^{2} G_{t, t^{v}}^{t, x ; \alpha_{\varepsilon}(v), v}\left[W_{2}\left(t^{v}, X_{t^{v}}^{t, x ; \alpha_{\varepsilon}(v), v}\right)\right]\right| \\
\leq & { }^{2} G_{t, t^{v}}^{t, x ; \alpha_{\varepsilon}(v), v}\left[W_{2}\left(t^{v}, X_{t^{v}}^{t, x ; u^{\varepsilon_{0}}, v^{\varepsilon_{0}}}\right)\right]+C \varepsilon_{1}+C \tau^{\frac{1}{2}} .
\end{aligned}
$$

Putting

$$
\Omega_{s}=\left\{{ }^{2} Y_{s}^{t, x ; u^{\varepsilon_{0}}, v^{\varepsilon_{0}}} \geq W_{2}\left(s, X_{s}^{t, x ; u^{\varepsilon_{0}}, v^{\varepsilon_{0}}}\right)-\varepsilon_{0}\right\}, s \in[t, T]
$$


we have

$$
\begin{aligned}
J_{2}\left(t, x ; \alpha_{\varepsilon}(v), v\right) & \leq{ }^{2} G_{t, t^{v}}^{t, x ; \alpha_{\varepsilon}(v), v}\left[\sum_{i=1}^{m} W_{2}\left(t_{i}, X_{t_{i}}^{t, x ; u^{\varepsilon_{0}}, v^{\varepsilon_{0}}}\right) 1_{\left\{t^{v}=t_{i}\right\}}\right]+C \varepsilon_{1}+C \tau^{\frac{1}{2}} \\
& \leq{ }^{2} G_{t, t^{v}}^{t, x ; \alpha_{\varepsilon}(v), v}\left[\sum_{i=1}^{m} W_{2}\left(t_{i}, X_{t_{i}}^{t, x ; u^{\varepsilon_{0}}, v^{\varepsilon_{0}}}\right) 1_{\left\{t^{v}=t_{i}\right\}} 1_{\Omega_{t_{i}}}\right]+C \varepsilon_{1}+C \tau^{\frac{1}{2}}+I,
\end{aligned}
$$

where

$$
I=\mid{ }^{2} G_{t, t^{v}}^{t, x ; \alpha_{\varepsilon}(v), v}\left[\sum_{i=1}^{m} W_{2}\left(t_{i}, X_{t_{i}}^{t, x ; u^{\varepsilon_{0}, v^{\varepsilon_{0}}}}\right) 1_{\left\{t^{v}=t_{i}\right\}}\right]-{ }^{2} G_{t, t^{v}}^{t, x ; \alpha_{\varepsilon}(v), v}\left[\sum_{i=1}^{m} W_{2}\left(t_{i}, X_{t_{i}}^{\left.t, x ; u^{\varepsilon_{0}, v^{\varepsilon_{0}}}\right)} 1_{\left\{t^{v}=t_{i}\right\}} 1_{\Omega_{t_{i}}}\right] \mid .\right.
$$

Since $\Phi_{2}, f_{2}$ and $h_{2}$ are bounded, from Lemma 2.2 we conclude that $W_{2}$ is bounded. Therefore,

$$
\begin{aligned}
I & \leq \mathbb{E}\left[\sum_{i=1}^{m}\left|W_{2}\left(t_{i}, X_{t_{i}}^{t, x ; u^{\varepsilon_{0}}, v^{\varepsilon_{0}}}\right)\right|^{2} 1_{\left\{t^{v}=t_{i}\right\}} 1_{\Omega_{t_{i}}} \mid \mathcal{F}_{t}\right]^{\frac{1}{2}} \\
& \leq C \sum_{i=1}^{m} \mathbb{P}\left(\Omega_{t_{i}}^{c} \mid \mathcal{F}_{t}\right)^{\frac{1}{2}} \leq C m \varepsilon_{0}^{\frac{1}{2}},
\end{aligned}
$$

where we have used (6.1) for the latter estimate. From the Lemmas 2.3, 2.4 and (6.7) we have

$$
\begin{aligned}
& { }^{2} G_{t, t^{v}}^{t, x ; \alpha_{\varepsilon}(v), v}\left[\sum_{i=1}^{m} W_{2}\left(t_{i}, X_{t_{i}}^{t, x ; u^{\varepsilon_{0}}, v^{\varepsilon_{0}}}\right) 1_{\left\{t^{v}=t_{i}\right\}} 1_{\Omega_{t_{i}}}\right] \leq{ }^{2} G_{t, t^{v}}^{t, x ; \alpha_{\varepsilon}(v), v}\left[\sum_{i=1}^{m}\left({ }^{2} Y_{t_{i}}^{t, x ; u^{\varepsilon_{0}}, v^{\varepsilon_{0}}}+\varepsilon_{0}\right) 1_{\left\{t^{v}=t_{i}\right\}} 1_{\Omega_{t_{i}}}\right] \\
& \leq{ }^{2} G_{t, t^{v}}^{t, x ; \alpha_{\varepsilon}}(v), v\left[\sum_{i=1}^{m}{ }^{2} Y_{t_{i}}^{t, x ; u^{\varepsilon_{0}}, v^{\varepsilon_{0}}} 1_{\left\{t^{v}=t_{i}\right\}} 1_{\Omega_{t_{i}}}+\varepsilon_{0}\right] \\
& \leq{ }^{2} G_{t, t^{v}}^{t, x ; \alpha_{\varepsilon}}(v), v\left[\sum_{i=1}^{m}{ }^{2} Y_{t_{i}}^{t, x ; u^{\varepsilon_{0}}, v^{\varepsilon_{0}}} 1_{\left\{t^{v}=t_{i}\right\}} 1_{\Omega_{t_{i}}}\right]+C \varepsilon_{0},
\end{aligned}
$$

and using the above arguments we also have

$$
\left|{ }^{2} G_{t, t^{v}}^{t, x ; \alpha_{\varepsilon}(v), v}\left[\sum_{i=1}^{m}{ }^{2} Y_{t_{i}}^{t, x ; u^{\varepsilon_{0}}, v^{\varepsilon_{0}}} 1_{\left\{t^{v}=t_{i}\right\}} 1_{\Omega_{t_{i}}}\right]-{ }^{2} G_{t, t v}^{t, x ; \alpha_{\varepsilon}(v), v}\left[\sum_{i=1}^{m}{ }^{2} Y_{t_{i}}^{t, x ; \alpha_{\varepsilon}(v), v} 1_{\left\{t^{v}=t_{i}\right\}}\right]\right| \leq C m \varepsilon_{0}^{\frac{1}{2}} .
$$

Consequently,

$$
\begin{aligned}
& { }^{2} G_{t, t^{v}}^{t, x ; \alpha_{\varepsilon}(v), v}\left[\sum_{i=1}^{m} W_{2}\left(t_{i}, X_{t_{i}}^{t, x ; u^{\varepsilon_{0}}, v^{\varepsilon_{0}}}\right) 1_{\left\{t^{v}=t_{i}\right\}} 1_{\Omega_{t_{i}}}\right] \\
\leq & { }^{2} G_{t, t^{v}}^{t, x ; \alpha_{\varepsilon}(v), v}\left[{ }^{2} Y_{t^{v}}^{t, x ; u^{\varepsilon_{0}}, v^{\varepsilon_{0}}}\right]+C \varepsilon_{0}+C m \varepsilon_{0}^{\frac{1}{2}} \\
\leq & \left|{ }^{2} G_{t, t^{v}}^{t, x ; \alpha_{\varepsilon}}(v), v\left[{ }^{2} Y_{t^{v}}^{t, x ; u^{\varepsilon_{0}}, v^{\varepsilon_{0}}}\right]-{ }^{2} G_{t, t^{v}}^{t, x ; u^{\varepsilon_{0}}, v^{\varepsilon_{0}}}\left[{ }^{2} Y_{t^{v}}^{t, x ; u^{\varepsilon_{0}}, v^{\varepsilon_{0}}}\right]\right| \\
& +{ }^{2} G_{t, t^{v}}^{t, x ; u^{\varepsilon_{0}}, v^{\varepsilon_{0}}}\left[{ }^{2} Y_{t^{v}}^{t, x ; u^{\varepsilon_{0}}, v^{\varepsilon_{0}}}\right]+C \varepsilon_{0}+C m \varepsilon_{0}^{\frac{1}{2}} \\
= & \left|{ }^{2} G_{t, t^{v}}^{t, x ; \alpha_{\varepsilon}}(v), v\left[{ }^{2} Y_{t^{v}}^{t, x ; u^{\varepsilon_{0}}, v^{\varepsilon_{0}}}\right]-{ }^{2} G_{t, t^{v}}^{t, x ; u^{\varepsilon_{0}}, v^{\varepsilon_{0}}}\left[{ }^{2} Y_{t^{v}}^{\left.t, x ; u^{\varepsilon_{0}}, v^{\varepsilon_{0}}\right]}\right]\right| \\
& +J_{2}\left(t, x ; u^{\varepsilon_{0}}, v^{\varepsilon_{0}}\right)+C \varepsilon_{0}+C m \varepsilon_{0}^{\frac{1}{2}} \\
\leq & J_{2}\left(t, x ; u^{\varepsilon_{0}}, v^{\varepsilon_{0}}\right)+C \varepsilon_{0}+C m \varepsilon_{0}^{\frac{1}{2}}+C \tau^{\frac{1}{2}},
\end{aligned}
$$


where we have used the fact that

$$
\left|{ }^{2} G_{t, t^{v}}^{t, x ; \alpha_{\varepsilon}(v), v}\left[{ }^{2} Y_{t^{v}}^{t, x ; u^{\varepsilon_{0}}, v^{\varepsilon_{0}}}\right]-{ }^{2} G_{t, t^{v}}^{t, x ; u^{\varepsilon_{0}}, v^{\varepsilon_{0}}}\left[{ }^{2} Y_{t^{v}}^{t, x ; u^{\varepsilon_{0}}, v^{\varepsilon_{0}}}\right]\right| \leq C \tau^{\frac{1}{2}} .
$$

Indeed, let us consider the following BSDE

$$
\left\{\begin{array}{l}
y_{s}={ }^{2} Y_{t^{v}}^{t, x ; u^{\varepsilon_{0}}, v^{\varepsilon_{0}}}+\int_{s}^{t^{v}} f_{2}\left(r, X_{r}^{t, x ; \alpha_{\varepsilon}(v), v}, y_{r}, z_{r}, \alpha_{\varepsilon}\left(v_{r}\right), v_{r}\right) \mathrm{d} r+k_{t^{v}}-k_{s}-\int_{s}^{t^{v}} z_{r} \mathrm{~d} B_{r} \\
y_{s} \geq h_{2}\left(s, X_{s}^{t, x ; \alpha_{\varepsilon}(v), v}\right), \quad s \in\left[t, t^{v}\right] \\
k_{t}=0, \quad \int_{t}^{t^{v}}\left(y_{r}-h_{2}\left(r, X_{r}^{t, x ; \alpha_{\varepsilon}(v), v}\right)\right) d k_{r}=0
\end{array}\right.
$$

which compared with

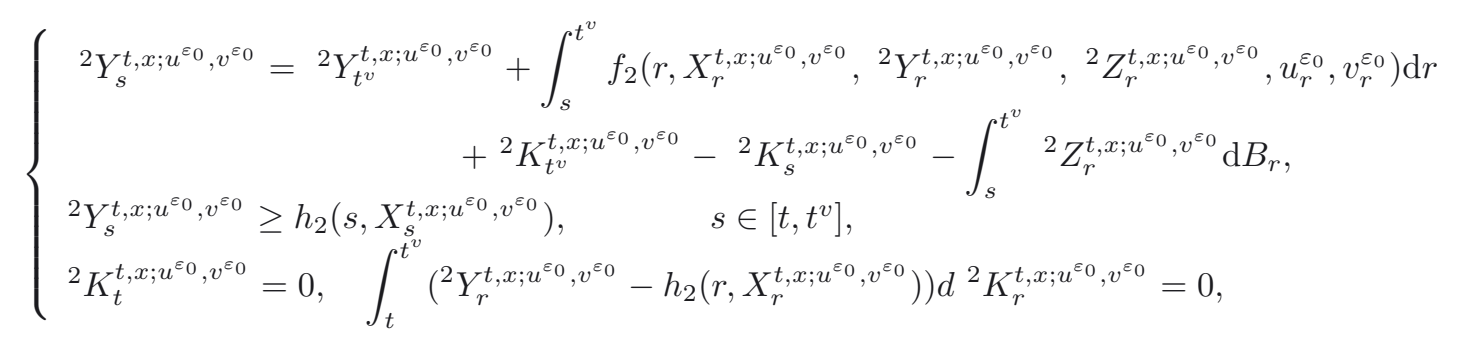

Note that $\alpha_{\varepsilon}(v)=u^{\varepsilon_{0}}$, on $\left[\left[t, t^{v}\right]\right], v=v^{\varepsilon_{0}}$, on $\left[\left[t, S^{v}\right]\right]$, and from Lemma 2.3 we obtain

$$
\begin{aligned}
& \left|{ }^{2} G_{t, t^{v}}^{t, x ; \alpha_{\varepsilon}(v), v}\left[{ }^{2} Y_{t^{v}}^{t, x ; u^{\varepsilon_{0}}, v^{\varepsilon_{0}}}\right]-{ }^{2} G_{t, t^{v}}^{t, x ; u^{\varepsilon_{0}}, v^{\varepsilon_{0}}}\left[{ }^{2} Y_{t^{v}}^{t, x ; u^{\varepsilon_{0}}, v^{\varepsilon_{0}}}\right]\right|^{2} \\
\leq & C \mathbb{E}\left[\int_{t}^{t^{v}}\left|f_{2}\left(r, X_{r}^{t, x ; \alpha_{\varepsilon}(v), v}, y_{r}, z_{r}, \alpha_{\varepsilon}(v)_{r}, v_{r}\right) \mathrm{d} r-f_{2}\left(r, X_{r}^{t, x ; u^{\varepsilon_{0}}, v^{\varepsilon_{0}}}, y_{r}, z_{r}, u_{r}^{\varepsilon_{0}}, v_{r}^{\varepsilon_{0}}\right)\right|^{2} \mid \mathcal{F}_{t}\right] \\
& +C \mathbb{E}\left[\sup _{r \in\left[t, t^{v}\right]}\left|h_{2}\left(r, X_{r}^{t, x ; \alpha_{\varepsilon}(v), v}\right)-h_{2}\left(r, X_{r}^{t, x ; u^{\varepsilon_{0}}, v^{\varepsilon_{0}}}\right)\right|^{2} \mid \mathcal{F}_{t}\right]^{\frac{1}{2}} \\
= & C \mathbb{E}\left[\int_{S_{v}^{v}}^{t^{v}}\left|f_{2}\left(r, X_{r}^{t, x ; \alpha_{\varepsilon}(v), v}, y_{r}, z_{r}, \alpha_{\varepsilon}(v)_{r}, v_{r}\right) \mathrm{d} r-f_{2}\left(r, X_{r}^{t, x ; u^{\varepsilon_{0}}, v^{\varepsilon_{0}}}, y_{r}, z_{r}, u_{r}^{\varepsilon_{0}}, v_{r}^{\varepsilon_{0}}\right)\right|^{2} \mid \mathcal{F}_{t}\right] \\
& +C \mathbb{E}\left[\sup _{r \in\left[S^{v}, t^{v}\right]}\left|X_{r}^{t, x ; \alpha_{\varepsilon}(v), v}-X_{r}^{t, x ; u^{\varepsilon_{0}}, v^{\varepsilon_{0}}}\right|{ }^{2} \mid \mathcal{F}_{t}\right]^{\frac{1}{2}} \\
\leq & C \mathbb{E}\left[\int_{S^{v}}^{t^{v}} 1_{\left\{v_{r} \neq v_{r}^{\varepsilon_{0}}\right\}} \mid \mathcal{F}_{t}\right]+C \tau^{\frac{1}{2}} \leq C \mathbb{E}\left[t^{v}-S^{v} \mid \mathcal{F}_{t}\right]+C \tau^{\frac{1}{2}} \leq C \tau^{\frac{1}{2}},
\end{aligned}
$$

where we have used the boundedness of $f_{2}, b$ and $\sigma$. Consequently,

$$
{ }^{2} G_{t, t^{v}}^{t, x ; \alpha_{\varepsilon}(v), v}\left[\sum_{i=1}^{m} W_{2}\left(t_{i}, X_{t_{i}}^{t, x ; u^{\varepsilon_{0}, v^{\varepsilon_{0}}}}\right) 1_{\left\{t^{v}=t_{i}\right\}} 1_{\Omega_{t_{i}}}\right] \leq C \tau^{\frac{1}{2}}+J_{2}\left(t, x ; u^{\varepsilon_{0}}, v^{\varepsilon_{0}}\right)+C \varepsilon_{0}+C m \varepsilon_{0}^{\frac{1}{2}},
$$

and thus, (6.8) and (6.9) yield

$$
J_{2}\left(t, x ; \alpha_{\varepsilon}(v), v\right) \leq J_{2}\left(t, x ; u^{\varepsilon_{0}}, v^{\varepsilon_{0}}\right)+C \varepsilon_{0}+C m \varepsilon_{0}^{\frac{1}{2}}+C \varepsilon_{1}+C \tau^{\frac{1}{4}} .
$$

We can choose $\tau>0, \varepsilon_{0}>0$, and $\varepsilon_{1}>0$ such that $C \varepsilon_{0}+C m \varepsilon_{0}^{\frac{1}{2}}+C \varepsilon_{1}+C \tau^{\frac{1}{4}} \leq \varepsilon$ and $\varepsilon_{0}<\varepsilon$. Thus,

$$
J_{2}\left(t, x ; \alpha_{\varepsilon}(v), v\right) \leq J_{2}\left(t, x ; u^{\varepsilon_{0}}, v^{\varepsilon_{0}}\right)+\varepsilon, v \in \mathcal{V}_{t, T} .
$$


Using a symmetric argument we can construct $\beta_{\varepsilon} \in \mathcal{B}_{t, T}$ such that, for all $u \in \mathcal{U}_{t, T}$,

$$
J_{1}\left(t, x ; u, \beta_{\varepsilon}(u)\right) \leq J_{1}\left(t, x ; u^{\varepsilon_{0}}, v^{\varepsilon_{0}}\right)+\varepsilon, \quad \beta_{\varepsilon}\left(u^{\varepsilon_{0}}\right)=v^{\varepsilon_{0}} .
$$

Finally, from (6.5), (6.10), (6.2) and Lemma 5.2 it follows that $\left(\alpha_{\varepsilon}, \beta_{\varepsilon}\right)$ satisfies Definition 5.1. Hence, $\left(e_{1}, e_{2}\right)$ is a Nash equilibrium payoff.

Acknowledgements. The author thanks two anonymous referees for their careful reading and helpful suggestions.

\section{REFERENCES}

[1] R. Buckdahn, P. Cardaliaguet and M. Quincampoix, Some recent aspects of differential game theory. Dynamic Games Appl. 1 (2011) $74-114$

[2] R. Buckdahn, P. Cardaliaguet and C. Rainer, Nash equilibrium payoffs for nonzero-sum Stochastic differential games. SIAM J. Control Optim. 43 (2004) 624-642.

[3] R. Buckdahn and J. Li, Stochastic differential games and viscosity solutions of Hamilton-Jacobi-Bellman-Isaacs equations. arXiv:math/0702131.

[4] R. Buckdahn and J. Li, Stochastic differential games with reflection and related obstacle problems for Isaacs equations arXiv:0707.1133.

[5] N. El Karoui, C. Kapoudjian, E. Pardoux, S. Peng and M.C. Quenez, Reflected solutions of backward SDE's, and related obstacle problems for PDE's. Ann. Probab. 25 (1997) 702-737.

[6] N. El Karoui, S. Peng and M.C. Quenez, Backward stochastic differential equation in finance. Math. Finance 7 (1997) 1-71.

[7] W.H. Fleming, P.E. Souganidis, On the existence of value functions of twoplayer, zero-sum stochastic differential games. Indiana Univ. Math. J. 38 (1989) 293-314.

[8] S. Hamadène, J. Lepeltier and A. Matoussi, Double barrier backward SDEs with continuous coefficient. In Backward Stochastic Differential Equations. Pitman Res. Notes Math. Ser., vol. 364. Edited by El Karoui Mazliak (1997) 161-175.

[9] Q. Lin, A BSDE approach to Nash equilibrium payoffs for stochastic differential games with nonlinear cost functionals. Stochastic Process. Appl. 122 (2012) 357-385.

[10] Q. Lin, Nash equilibrium payoffs for stochastic differential games with jumps and coupled nonlinear cost functionals. arXiv: $1108.3695 \mathrm{v} 1$.

[11] S. Peng, Backward stochastic differential equations-stochastic optimization theory and viscosity solutions of HJB equations, in Topics Stoch. Anal., edited by J. Yan, S. Peng, S. Fang and L. Wu., Ch. 2 (Chinese vers.) (1997).

[12] Z. Wu and Z. Yu, Dynamic programming principle for one kind of stochastic recursive optimal control problem and HamiltonJacobi-Bellman equation. arXiv:0704.3775. 